\author{
Researcher Aurelian-Petrus PLOPEANU, PhD \\ E-mail: aplopeanu@gmail.com \\ "Alexandru Ioan Cuza" University of Iasi (UAIC) \\ Researcher Daniel HOMOCIANU, PhD \\ E-mail: dan.homocianu@gmail.com \\ Department of Research, Faculty of Economics and \\ Business Administration (FEAA), UAIC \\ Professor Dinu AIRINEI, PhD \\ E-mail: adinu@uaic.ro \\ Department of Accounting, Business Information \\ Systems and Statistics, FEAA, UAIC
}

\title{
DETERMINANTS OF ENTREPRENEURIAL PROCLIVITY OF STUDENTS IN ECONOMICS AND BUSINESS ADMINISTRATION
}

\begin{abstract}
This paper's purpose is to validate a set of characteristics concerning Romanian students in economics and business administration regarding their entrepreneurial proclivity after graduation, based on a questionnaire survey with a considerable number of responses and categories of variables. The empirical analyses are based on multinomial logistic regressions, while several tests were used to check the robustness and verify the accuracy of the models - AUROC diagnostic test value of 0.84. We found a strong new influence of the paternal role models towards the students' proclivity to be self-employed after graduation, especially from father to daughter. Moreover, the results confirm the fundamental influence of personality traits, gender and personal ethical beliefs. The article also offers further possibilities of top feature extraction for some job categories considered based on standardized coefficients.

Keywords: self-employed (SE); parental role models (PRMs); openness; ethical beliefs; empirical analysis, multinomial logistic regressions (MNL).

JEL classification: L26, B23, Y10

1. Introduction

Generally, the intentions to become an entrepreneur are based on complex sets of personality traits, familial legacies, and economic beliefs, educational and cultural mixtures. There is a major interest among the students to become their own bosses after graduation. A research conducted on 765 students in terminal year from several Romanian universities stressed the same trend. Those students who studied economic disciplines manifested an entrepreneurial appetite (52\% of them) after graduation, rather than being employed (57\% of them). Also, the same research found that $52 \%$ of them would prefer to start their own business in the next 2 years (Matei et al. 2014, 3-5). This is of high concern, given the fact that, on
\end{abstract}

DOI: $10.24818 / 18423264 / 52.3 .18 .07$ 
one side, there is an improvement of perception among the society towards entrepreneurial (private) initiatives (Lupea et al. 2016, 14), while, on the other side, the young people that may have these kind of initiatives are leaving Romania, as shown in another research using data from the National Trade Register Office ONRC.

The importance of the entrepreneurship processes on economic growth has been highly emphasized in the specialized literature (Acs and Szerb 2009). Consequently, we aim to estimate whether the Romanian students' entrepreneurship intentions after graduation are just a matter of external incentives through learning, training and choosing a faculty which offers a business and economics curriculum, or there is much more than this, namely the "internal codes" which are usually observed in their parents' behaviors, making these intentions a complex of familial, behavioral, ethical and educational determinants.

Given the short-term capitalist tradition in Romania, manifested especially after the fall of communism and the academic one, which, in terms of entrepreneurship courses, follows the same evolution, this paper is an important landmark for further improvements and adaptations of the academic courses to the student's specificities. The implication of this article for policy makers is also considerable because of a greater understanding of the students' proclivity towards entrepreneurship in the context of an unstable Romanian economy, with an unpredictable future, which still leads to high levels of migration of young and skilled workers.

The reasons why we have chosen the North-Eastern (NE) region of Romania are based on the following considerations. First of all, these are communities with the lowest living standards compared to other Romanian regions. The entrepreneurial initiatives are growing in these regions, according to ONRC data on the registration of natural and legal persons. We also have to keep in mind that Iasi is the largest city and also the most important university and business center in this part of Romania, attracting many students to settle here after graduation. Attractive future careers, higher wages and a vibrant social life are elements that highlight Iasi as a main urban center. Furthermore, the preference for investigating students attending Faculty of Economics and Business Administration (FEAA) is not based on convenience in terms of how data are collected, but on the specificity of this educational institution. After all, FEAA can be seen as a place that brings together students studying economic disciplines, therefore, closest to entrepreneurship, both in terms of the specificity of the disciplines and the individual vocation. After analyzing the entrepreneurship intentions among the students currently attending FEAA, this paper could help us understanding the potential factors that may influence a student's decision to start a new business after graduation.

Firstly, our study adds to the existing entrepreneurship literature the influence of the individuals' economic beliefs, their educational performances (high-school and baccalaureate grades) and personality traits, the familial 


\section{Determinants of Entrepreneurial Proclivity of Students in Economics and Business Administration}

background, involving the possibility of intergenerational transfer of entrepreneurial traits, and the religious beliefs for better explaining the entrepreneurial propensity of students currently attending FEAA. Secondly, our paper adds to the literature the role of personality traits and individual religiosity in influencing the intentions towards entrepreneurship. To our knowledge, this article is among the first ones to investigate the relationship between SE parental background, personality traits and individual religiosity and the entrepreneurial intentions among the students attending a targeted Romanian faculty. Thirdly, we emphasize not only the paternal role models as the core of our article, but also the maternal ones, while highlighting the differences between these categories both in terms of significance and coefficients. Equally interesting is another finding further discussed in our paper, that intergenerational transfer of entrepreneurial / SE behaviors from parents, especially from the fathers, is received differently by the descendants, depending on their gender. Fourthly, another idea from our paper that augments the findings already made in the field of entrepreneurial literature (Chlosta et al. 2012) is that the individual's personality traits, especially openness, are an important element that interferes with one's decision to choose a SE career after graduation. Many articles have analyzed the influence that personality traits have on the decision to become SE (Rauch and Frese 2007), but no explanation has been given as to why some people choose differently than others in relation to their future profession. We have uses the openness variable to better explain why.

\section{Literature review}

In order to provide an in-depth explanation on why the PRMs determine their offspring to choose to become SE after graduation, we have to consider several variables that may draw a comprehensive scenario regarding the future decision of becoming an entrepreneur. They are categorized and described as it follows.

First, the way in which families may influence the entrepreneurial outcomes received some evidence in the literature. Moreover, the relationship between the parental entrepreneurship and the propensity a child could have to become an entrepreneur was also analysed (Corak and Piraino 2011). The parental influence towards students' propensity for entrepreneurship is highly demonstrated. Obviously, it is considered that students with parental-type businesses are more likely to manifest entrepreneurial intentions after graduation (Gevrek and Gevrek 2010, 591). Thomas Dunn and Douglas Holtz-Eakin (2000, 283) emphasized that a son's probability to become SE increases by $200 \%$ when one of his parents is also SE. Other ideas stressed an interesting motivation for the transfer of entrepreneurial background from parents to their children, to be more specific, those parents who are involved in entrepreneurial activities could transmit their appetite for this kind of economic behaviors to their children using the role models (Chlosta et al. 2012).

The personality traits have an important influence towards decision-making processes (Chlosta et al. 2012, 122-125). In our models, we take into consideration

DOI: $10.24818 / 18423264 / 52.3 .18 .07$ 
Aurelian-Petrus Plopeanu, Daniel Homocianu, Dinu Airinei

conscientiousness, openness and agreeableness, the best suited characteristics for our story. The first element, conscientiousness, is about individuals who are competent, strong-willed, with an excellent capacity to systematically fulfill all the obligations taken into account with great accent put on self-discipline, determination and reliability (Judge, Martocchio and Thoresen 1997, 747). Openness is the second personality trait, a human behavior leaning on how openminded an individual really is and how easy he adopts new ideas (Njoku, Ebeh, and Mbaeri 2017, 10), denoting flexibility, creativity and initiative spirit. The third personality feature is agreeableness and a person with this quality is characterized by a great sense of altruism, willing to help the others in need (Rothmann and Coetzer 2003, 69). Religion is an important variable that may influence the entrepreneurship process and outcomes, especially in the context of today's globalization and multiculturalism. Moral and spiritual values from a religious commitment are considered extremely needed in a public sector workplace. More, the individuals who work in the public sector are animated in greater degrees than their counterparts from the private sector by the intrinsic rewards of work compared to the extrinsic ones (Houston and Cartwright 2017, 91). In conclusion, the extrinsic rewards (e.g. higher salary) don't motivate the public employees the way the intrinsic ones could do (Frank and Lewis 2004), therefore letting us to consider that the spiritual or religious motivations play a more important role for them than the economic or material rewards.

The students in economics and business administration with higher averages of baccalaureate grades are more likely to choose to work as SE or in small and medium-sized enterprises (SMEs) and large private companies (LPCs) rather than in the public sector. The explanation stresses that meritocratic features of the labor force who graduated high school, primarily employed in the private sector.

The relationship between trust and entrepreneurship is emphasized with great interest in the literature. Francis Fukuyama (1996) stressed the concept of high-trust environment as a collection of 'trust clusters' where the relationships between known / unknown individuals are based on the support of trust. The effect of such societal settings positively contributes to entrepreneurship, while a lowtrust environment disfavours any individual entrepreneurial initiatives. Among possible explanations why in some societies there is still low-trust institutional milieux, there are some solid arguments: the cultural or historical path dependencies, the legacies of "real socialism" in post-communist societies that eroded the social norms.

Competition is an important ingredient for economic growth. The stimulating role played by competition, entrepreneurship, innovation and firm start-ups on the economic growth is interesting to highlight (North and Thomas 1973). It is considered that numerous and complex institutional arrangements, which may include also the elements belonging to what we call legal institutions, may generate specific stimulus for the entrepreneurship process, with different effects on the economic growth (Palagashvili 2015, 14). 


\section{Determinants of Entrepreneurial Proclivity of Students in Economics and Business Administration}

Migration and entrepreneurship are a nexus that is important for our study. The decision to migrate after graduation is seen as a possibility to accept and take more risks than usual (Neville, Orser, Riding, and Jung 2014), quite close as manifestation as the entrepreneurship processes. Moreover, Brixy, Sternberg, and Vorderwlbecke (2013) stressed that immigrants are more oriented towards SE professions due to their exclusion from more formal wage opportunities.

Individual freedom is an institutional framework that stimulates entrepreneurship and, therefore, economic growth (North and Thomas 1973). The value of labor is an essential element that stimulates the economic growth and is influenced greatly by culture through personal associated beliefs. Moreover, successful entrepreneurship specifically involves the entrepreneur's positive attitude towards the value of hard work to achieve long term objectives (Lee and Lee 2015, 897).

\section{Data description and methodology}

During the winter of 2016-2017, spanning over a period of four months, we have conducted a questionnaire-based investigation within Alexandru Ioan Cuza University of Iasi. We have focused entirely on the students from FEAA. To ensure the statistical relevance, we have designed a proper size of the sample by considering a statistical population of 7093 students enrolled in 2017. We have collected 1100 unique responses/records for 1155 distributed questionnaires. We assured students of the confidentiality of their answers. Since we applied printed questionnaires, we controlled the received responses and that is why the response rate was higher than $95 \%$. The catchment area for our survey was represented by the Eastern and NE regions of Romania.

The survey followed a deep understanding of such a phenomenon among the students from FEAA. It results may encourage greatly the entrepreneurial intentions. This deep understanding of such a complex decision was based on the extensive deforestation of the relevant literature on the students' entrepreneurship initiatives.

The dependent categorical variable job_type has 4variantsregarding the question "Where would you like to work after graduation?"about post-graduation professional careers: SE (1); employee in SMEs (2); employee in LPCs (3) and in public / state companies and institutions (PCIs), coded as 4.

In Table 1, we have synthesized the independent variables used in this study and associated with corresponding questions and responses.

In order to analyze the determinant factors that influence the probability (Scott Long and Jeremy Freese 2006) to choose a certain job category (outcome), we have used the following empirical model (eq.1):

$$
\ln \left[\operatorname{Pr}_{\mathrm{i}}(\mathrm{K} \mid \mathrm{X}) / \operatorname{Pr}_{\mathrm{i}}(\operatorname{ref} \mid \mathrm{X})\right]=\beta_{0, \mathrm{~K} \mid \text { ref }}+\beta_{1, \mathrm{~K} \mid \text { ref }} * \text { SeJobAtLeast } 1 \mathrm{P}_{\mathrm{i}}+\sum_{j=2}^{m} \beta_{\mathrm{j}, \mathrm{K} \text { |ref }} * \mathrm{X}_{\mathrm{ij}}+\varepsilon_{\mathrm{K} \text { |ref } \mathrm{i}}
$$

Table 1. Summary statistics including some abbreviations of the variables used.

DOI: $10.24818 / 18423264 / 52.3 .18 .07$ 
Aurelian-Petrus Plopeanu, Daniel Homocianu, Dinu Airinei

\begin{tabular}{|c|c|c|c|}
\hline & Mean & Min & Max \\
\hline \multicolumn{4}{|l|}{ VAR I A B L E S } \\
\hline \multicolumn{4}{|l|}{ Probability of job_type as outcome variable } \\
\hline Probability of SE (value 1 ) & 0.2 & 0 & 1 \\
\hline Probability of employee in a SME (value 2) & 0.18 & 0 & 1 \\
\hline Probability of employee in a LPC (value 3) - "employee in a PCI" coded as 4 set as reference & 0.44 & 0 & 1 \\
\hline \multicolumn{4}{|l|}{ Individual's characteristics } \\
\hline Age of respondent (age) & 20.67 & 18 & 33 \\
\hline Gender (male) & 0.29 & 0 & 1 \\
\hline \multicolumn{4}{|l|}{ Individual's beliefs, personality traits and school performance } \\
\hline secular & 0.62 & 0 & 1 \\
\hline High believe in God (high_believe_god) & 0.77 & 0 & 1 \\
\hline Conscientiousness as hard work / sense of responsibility / perseverance (conscientiousness) & 0.92 & 0 & 1 \\
\hline Openness as independence / creativity / imagination (openness) & 0.6 & 0 & 1 \\
\hline Agreeableness as good manners / tolerance / obedience / altruism (agreeableness) & 0.95 & 0 & 1 \\
\hline Individual's intention of migration after graduation (migr_index) & 4.15 & 1 & 10 \\
\hline State welfare (state_welfare) & 0.41 & 0 & 1 \\
\hline State must involve more in economy (state_must_inv_more_in_ec) & 0.71 & 0 & 1 \\
\hline Competition stimulating effect (compet_stim_effect) & 0.9 & 0 & 1 \\
\hline Fear of competition (fear_of_compet) & 0.42 & 0 & 1 \\
\hline Labor is the source of success in life/society (labour_success_source) & 0.95 & 0 & 1 \\
\hline Individual freedom is a factor of progress (indiv_libert_progr_cause) & 0.71 & 0 & 1 \\
\hline Acceptance of undeserved financial advantages (undesr ${ }_{\text {_f }}$ in_advtg) & 0.08 & 0 & 1 \\
\hline Accepting the avoidance of buying public transport tickets (avoid_buy_pub_transp_tickds) & 0.09 & 0 & 1 \\
\hline Child needs family to harmoniously develop (child_needs_fam 2dev) & 0.97 & 0 & 1 \\
\hline Parents asume responsibility for children's future (p_asum_resp 4 child_future) & 0.88 & 0 & 1 \\
\hline Average of high-school grades (high_school_avg_grade) & 8.91 & 5.23 & 10 \\
\hline Average of baccalaureate grades (bac_avg_grade) & 8.27 & 6 & 10 \\
\hline \multicolumn{4}{|l|}{ Individual's trust } \\
\hline Interpersonal trust (interpers_trust $2, \ldots$, interpers_trust 5 -interpers_trust 1 as reference) & 2.41 & 1 & 5 \\
\hline Trust in government (trust_governm) & 0.09 & 0 & 1 \\
\hline Trust in church (trust_church) & 0.41 & 0 & 1 \\
\hline Trust no institution (trust_no_instit) - trust in other institutions as reference & 0.39 & 0 & 1 \\
\hline Do you trust the Romanian legal system (trust_in_legal_instit) - no as reference & 0.23 & 0 & 1 \\
\hline \multicolumn{4}{|l|}{ Family background } \\
\hline Number of siblings (no_sibling) & 0.21 & 0 & 1 \\
\hline Number of siblings (one_sibling) & 0.5 & 0 & 1 \\
\hline Number of siblings (two_siblings) - other cases as reference & 0.2 & 0 & 1 \\
\hline Family income - 1500-2499 RON (income2) & 0.31 & 0 & 1 \\
\hline Family income - 2500-3499 RON (income3) & 0.2 & 0 & 1 \\
\hline Family income - $3500-4499$ RON (income 4 ) & 0.14 & 0 & 1 \\
\hline Family income $-4500-5499 \mathrm{RON}$ (income 5) & 0.05 & 0 & 1 \\
\hline Family income - 5500-6499 RON (income 6) & 0.03 & 0 & 1 \\
\hline Family income - more than $6500 \mathrm{RON}$ (income7) - less than 1500 RON as reference & 0.06 & 0 & 1 \\
\hline Urban (urban) & 0.57 & 0 & 1 \\
\hline Proximity to Iasi (dist050_2lasi, dist50100_2lasi, dist100150_2lasi, other cases as reference & 82.3 & 0 & 552 \\
\hline \multicolumn{4}{|l|}{ Mother and father's education } \\
\hline Mother's low education meaning lower and lower secondary ( $m$ low) & 0.06 & 0 & 1 \\
\hline Mother's upper secondary education (m_upper_sec) - tertiary and other types of education as reference & 0.72 & 0 & 1 \\
\hline Father's low education meaning lower and lower secondary $\left(f_{-}\right.$low $)$ & 0.05 & 0 & 1 \\
\hline Father's upper secondary education (__upper_sec) - tertiary and other types of education as reference & 0.75 & 0 & 1 \\
\hline \multicolumn{4}{|l|}{ Parents' SE profession, faith in God and severity } \\
\hline SE profession - mother only (self_emp_only_m), eq.2 & 0.05 & 0 & 1 \\
\hline SE profession - father only (self_emp_only_f), eq. 2 & 0.11 & 0 & 1 \\
\hline SE profession - both (self_emp_both_p), eq.2 & 0.04 & 0 & 1 \\
\hline SE profession - at least one parent (self_emp_at_leastlp), eq. 1 - no parent as reference for both eq. $1 \& 2$ & 0.2 & 0 & 1 \\
\hline Parents' faith in God - mother only (faith_god_only_m) & 0.33 & 0 & 1 \\
\hline Parents' faith in God - father only (faith_god_only_f) & 0.03 & 0 & 1 \\
\hline Parents' faith in God - both (faith_god_both_p) - no parent as reference & 0.49 & 0 & 1 \\
\hline severity & 5.92 & 1 & 10 \\
\hline
\end{tabular}




\section{Determinants of Entrepreneurial Proclivity of Students in Economics and Business Administration}

$\operatorname{Pr}_{\mathrm{i}}$ from eq. 1 is the probability of a certain job category / outcome $\mathrm{K}$ for individual $\mathrm{i}$, where $\mathrm{K}=1,2,3$ and 4 is set as reference ( $\mathrm{ref}=4$ ), $\mathrm{i}=1, . ., \mathrm{n}, \mathrm{n}$ as the total number of responses and $\mathrm{j}=2, . ., \mathrm{m}, \mathrm{m}$ as the total number of independent variables. $\beta_{\mathrm{j}, \mathrm{K} \text { |ref }}$ measures the effect of a change in variable $X_{\mathrm{ij}}$ on the probability of choosing a certain category of jobs / professions. SeJobAtLeast $1 \mathrm{P}_{\mathrm{i}}$ is 1 if at least one parent in the student's family has a SE nature job.

The explanatory variable $X_{i j}$ (see eq.1) contains an array of the following individual and family characteristics: age, gender, income, individual beliefs (faith in God, the value of work, individual freedom, competition and state involvement as sources of progress in life/society, trust in institutions and in other people, attitude towards migration after graduation, child-parent relationship, ethical beliefs (the justification to receive undeserved financial advantages and avoid buying public transport tickets) and personality traits (conscientiousness, agreeableness and openness), individual education (average of high-school and baccalaureate grades), family wealth (proxied by monthly income) and size (number of siblings), residence address (proximity to the city of Iasi), parent's education, faith in God and severity. $\epsilon_{\mathrm{K} \mid r e f} \mathrm{i}$ is the error term.

$$
\begin{aligned}
& \ln \left[\operatorname{Pr}_{\mathrm{i}}(\mathrm{K} \mid \mathrm{X}) / \operatorname{Pr}_{\mathrm{i}}(\operatorname{ref} \mid \mathrm{X})\right]=\theta_{0, \mathrm{~K} \text { |ref }}+\theta_{1, \mathrm{~K} \text { |ref }} * \text { SeJobMotherOnly } \mathrm{y}_{\mathrm{i}}+\theta_{2, \mathrm{~K} \mid \text { ref }} * \text { SeJobFatherOnly }_{\mathrm{i}}+ \\
& \theta_{3, \mathrm{~K} \mid \text { ref }} * \operatorname{SeJobBoth}_{\mathrm{i}}+\sum_{j=4}^{m} \theta_{\mathrm{j}, \mathrm{K} \text { |ref }} * \mathrm{X}_{\mathrm{ij}}+\varepsilon_{\mathrm{K} \mid \text { rer } \mathrm{i}}
\end{aligned}
$$

In another specification (eq.2), we differentiate between respondents with only their mothers having a SE nature profession (SeJobMotherOnly), only their fathers (SeJobFatherOnly) and both (SeJobBoth) with this type of professions.

The entire statistical analysis was performed using Stata 13.064 bits MP (MultiProcessing / Parallel Edition).

\section{Empirical Results using MNL}

We have used Stata in twelve successive MNL scenarios, also referred as sets of specifications from (a) to (1), and we describe the corresponding results below (Tables 2-6, and Figs.1 and 2).Initially, we have included in the model (eq.1) only the variable self_emp_at_leastlp - scenario (a), which proves to be strong and significant along all 12 scenarios(see Table 2).The result confirms that having at least one parent SE, significantly increases the probability of a student in economics and business administration to become SE after graduation. After 12 scenarios, we can identify the negative effect of the following variables: no_sibling, trust_in_legal_instit, fear_of_compet and labour_success_source in association with the SE category. A positive effect towards the tendency to become SE after graduation manifest the variables male, high_school_avg_grade, openness and avoid_buy_pub_transp_tickts. 
Aurelian-Petrus Plopeanu, Daniel Homocianu, Dinu Airinei

Table 2. MNL coefficients ( $\mathrm{Ln}$ of odds ratios) for estimating the probability to fit the professional category corresponding to outcome 1 - SE (eq.1)

\begin{tabular}{|c|c|c|c|c|c|c|c|c|c|c|c|c|}
\hline $\begin{array}{l}\text { Probability of outcome1 } \\
\text { (SE) }\end{array}$ & (a) & (b) & (c) & (d) & (e) & (f) & (g) & (h) & (i) & (j) & (k) & (1) \\
\hline self_emp_at_leastlp & $\begin{array}{l}\text { 108**** } \\
(0.26)\end{array}$ & $\begin{array}{l}\mathbf{1 . 1 4 * * *} \\
(0.27)\end{array}$ & $\begin{array}{l}\mathbf{1 . 1 3 * * *} \\
(0.27) \\
\end{array}$ & $\begin{array}{l}\mathbf{1 0 7 * * *} \\
(0.28)\end{array}$ & $\begin{array}{l}\mathbf{1 0 2 * * *} \\
(0.28)\end{array}$ & $\begin{array}{l}\mathbf{0 . 8 5 * * *} \\
(0.30) \\
\end{array}$ & $\begin{array}{l}\mathbf{0 . 8 7 * * *} \\
(0.31) \\
\end{array}$ & $\begin{array}{l}\mathbf{0 . 8 9 * * *} \\
(0.31)\end{array}$ & $\begin{array}{l}0.93^{* * * *} \\
(0.31) \\
\end{array}$ & $\begin{array}{l}\mathbf{0 . 9 1 * * *} \\
(0.32) \\
\end{array}$ & $\begin{array}{l}0.86^{* * * *} \\
(0.32) \\
\end{array}$ & $\begin{array}{l}\mathbf{0 . 8 5 * * *} \\
(0.32) \\
\end{array}$ \\
\hline male & & $\begin{array}{l}\mathbf{1 . 1 4 * * * *} \\
(0.23)\end{array}$ & $\begin{array}{l}\mathbf{1 . 1 4 * * * *} \\
(0.23)\end{array}$ & $\begin{array}{l}\mathbf{1 . 2 8 * * * *} \\
(0.25)\end{array}$ & $\begin{array}{l}\mathbf{1 . 1 1 * * * *} \\
(0.26)\end{array}$ & $\begin{array}{l}\text { 1.06**** } \\
(0.27)\end{array}$ & $\begin{array}{l}\text { 1.11**** } \\
(0.28)\end{array}$ & $\begin{array}{l}\text { 1.12**** } \\
(0.28)\end{array}$ & $\begin{array}{l}\mathbf{1 0 6 * * *} \\
(0.28)\end{array}$ & $\begin{array}{l}\mathbf{1 . 0 3 * * * *} \\
(0.29)\end{array}$ & $\begin{array}{l}\text { 1.04**** } \\
(0.29)\end{array}$ & $\begin{array}{l}\mathbf{1 . 0 4 * * * *} \\
(0.29)\end{array}$ \\
\hline no_sibling & & $\begin{array}{l}\mathbf{0 . 8 3 * *} \\
(0.42) \\
\end{array}$ & $\begin{array}{l}-\mathbf{0 . 8 *} \\
(0.42) \\
\end{array}$ & $\begin{array}{l}\mathbf{- 0 . 9 8 * *} \\
(0.45) \\
\end{array}$ & $\begin{array}{l}\mathbf{- 1 2} * * * \\
(0.45) \\
\end{array}$ & $\begin{array}{l}-\mathbf{1 . 1 2} \mathbf{2}^{* * * *} \\
(0.45)\end{array}$ & $\begin{array}{l}\mathbf{1 2 1} * * * \\
(0.46) \\
\end{array}$ & $\begin{array}{l}-1.22^{* * * *} \\
(0.47)\end{array}$ & $\begin{array}{l}-\mathbf{1 . 1 7} 7^{* * * *} \\
(0.47)\end{array}$ & $\begin{array}{l}-\mathbf{1 . 2 1} * * * \\
(0.47)\end{array}$ & $\begin{array}{l}-\mathbf{1 . 2 9} * * * \\
(0.47)\end{array}$ & $\begin{array}{l}-1.27^{* * * *} \\
(0.48) \\
\end{array}$ \\
\hline high_school_avg_grade & & & & $\begin{array}{l}\text { 0.39** } \\
(0.19)\end{array}$ & $\begin{array}{l}\mathbf{0 . 4 1}^{* * *} \\
(0.19)\end{array}$ & $\begin{array}{l}\mathbf{0 . 4 3 * *} \\
(0.20)\end{array}$ & $\begin{array}{l}0.48^{\text {*** }} \\
(0.20)\end{array}$ & $\begin{array}{l}0.49 * * * \\
(0.20)\end{array}$ & $\begin{array}{l}0.5^{* * * *} \\
(0.20)\end{array}$ & $\begin{array}{l}0.52^{* * * *} \\
(0.20)\end{array}$ & $\begin{array}{l}0.5 * * \\
(0.21)\end{array}$ & $\begin{array}{l}0.5 * * \\
(0.21)\end{array}$ \\
\hline high_believe_god & & & & & $\begin{array}{l}-0.67 * * \\
(0.29) \\
\end{array}$ & $\begin{array}{l}-0.71^{* * *} \\
(0.30)\end{array}$ & $\begin{array}{l}-0.62^{* * *} \\
(0.31) \\
\end{array}$ & $\begin{array}{l}-0.59 * * \\
(0.31)\end{array}$ & $\begin{array}{l}-0.54^{*} \\
(0.31)\end{array}$ & $\begin{array}{l}-0.55^{*} \\
(0.32)\end{array}$ & $\begin{array}{l}-0.47 \\
(0.32)\end{array}$ & $\begin{array}{l}-0.46 \\
(0.32)\end{array}$ \\
\hline agreeableness & & & & & & $\begin{array}{l}-\mathbf{- 0 . 8 8 *} \\
(0.50)\end{array}$ & $\begin{array}{l}-\mathbf{0 . 7 6} \\
(0.50)\end{array}$ & $\begin{array}{l}-0.72 \\
(0.50)\end{array}$ & $\begin{array}{l}\mathbf{- 0 . 6 4} \\
(0.51)\end{array}$ & $\begin{array}{l}-\mathbf{- 0 . 6 3} \\
(0.51)\end{array}$ & $\begin{array}{l}-\mathbf{- 0 . 5 9} \\
(0.51)\end{array}$ & $\begin{array}{l}-0.58 \\
(0.51)\end{array}$ \\
\hline openness & & & & & & $\begin{array}{l}\mathbf{0 . 4 9 * *} \\
(0.24) \\
\end{array}$ & $\begin{array}{l}\mathbf{0 . 4 7 *} \\
(0.25)\end{array}$ & $\begin{array}{l}0.44^{*} \\
(0.25)\end{array}$ & $\begin{array}{l}\mathbf{0 . 4 2 *} \\
(0.25)\end{array}$ & $\begin{array}{l}0.46^{*} \\
(0.25) \\
\end{array}$ & $\begin{array}{l}0.47^{*} \\
(0.26)\end{array}$ & $\begin{array}{l}\mathbf{0 . 4 8 *} \\
(0.26)\end{array}$ \\
\hline trust_in_legal_instit & & & & & & & $\begin{array}{l}-0.8 \text { **** } \\
(0.26)\end{array}$ & $\begin{array}{l}-0.77 * * * * \\
(0.26)\end{array}$ & $\begin{array}{l}-0.78^{* * * *} \\
(0.27)\end{array}$ & $\begin{array}{l}-0.8 \text { **** } \\
(0.27)\end{array}$ & $\begin{array}{l}-\mathbf{- 0 . 8 1} \text { ***k } \\
(0.27)\end{array}$ & $\begin{array}{l}-\mathbf{0 . 8 1} \text { ***k } \\
(0.27)\end{array}$ \\
\hline$\overline{\text { migr_index }}$ & & & & & & & & $\begin{array}{l}0.09 \\
(0.06)\end{array}$ & $\begin{array}{l}\mathbf{0 . 1}^{*} \\
(0.06)\end{array}$ & $\begin{array}{l}\mathbf{0}^{\mathbf{0}} \\
(0.06)\end{array}$ & $\begin{array}{l}\mathbf{0 . 0 8} \\
(0.06)\end{array}$ & $\begin{array}{l}\mathbf{0 . 0 8} \\
(0.06)\end{array}$ \\
\hline fear_of_compet & & & & & & & & & $\begin{array}{l}-0.55 * * \\
(0.23) \\
\end{array}$ & $\begin{array}{l}-0.56^{* * * *} \\
(0.23)\end{array}$ & $\begin{array}{l}\mathbf{- 0 . 5 6 * *} \\
(0.23) \\
\end{array}$ & $\begin{array}{l}-0.56^{* *} \\
(0.23) \\
\end{array}$ \\
\hline Iabor_success_source & & & & & & & & & & $\begin{array}{l}-\mathbf{1 6 2 * * *} \\
(0.62)\end{array}$ & $\begin{array}{l}-\mathbf{- 1 . 6 2 * * *} \\
(0.62)\end{array}$ & $\begin{array}{l}\mathbf{x}^{*} \mathbf{- 1 . 6 3 * * * *} \\
(0.62) \\
\end{array}$ \\
\hline avoid_buy_pub_transp_ & tickts & & & & & & & & & & $\begin{array}{l}\mathbf{1 . 0 2 * * * *} \\
(0.41)\end{array}$ & $\begin{array}{l}\mathbf{1 . 0 2 * * *} \\
(0.41) \\
\end{array}$ \\
\hline observations & $\mathbf{1 1 0 0}$ & 1100 & 1100 & 1038 & 1038 & 1038 & 1038 & 1038 & 1038 & 1038 & 1036 & 1036 \\
\hline $\begin{array}{l}\text { F-stat } \\
\text { [p-value] }\end{array}$ & $\begin{array}{l}\mathbf{1 8 . 7 4} \\
{[0.0003]} \\
\end{array}$ & $\begin{array}{l}84.53 \\
{[0.0022]} \\
\end{array}$ & $\begin{array}{l}\mathbf{9 3 . 5 3} \\
{[0.0036]}\end{array}$ & $\begin{array}{l}119.63 \\
{[0.0001]}\end{array}$ & $\begin{array}{l}138.39 \\
{[0.0001]}\end{array}$ & $\begin{array}{l}\mathbf{1 5 7 . 6 1} \\
{[0.0000]}\end{array}$ & $\begin{array}{l}195.05 \\
{[0.0000]}\end{array}$ & $\begin{array}{l}201.22 \\
{[0.0000]}\end{array}$ & $\begin{array}{l}219.47 \\
{[0.0000]}\end{array}$ & $\begin{array}{l}233.7 \\
{[0.0000]}\end{array}$ & $\begin{array}{l}246.29 \\
{[0.0000]}\end{array}$ & $\begin{array}{l}249.35 \\
{[0.0000]} \\
\end{array}$ \\
\hline Pseudo R square & 0.007 & 0.031 & 0.035 & 0.048 & 0.057 & 0.065 & 0.083 & 0.086 & 0.095 & 0.103 & 0.11 & 0.111 \\
\hline
\end{tabular}

Note 1: Standard errors in parentheses indicate significance at $10 \%(*), 5 \%(* *)$ and $1 \%(* * *)$, respectively.

Note 2: All sets of specifications including the background controls are listed below: (a) is the model's core (self_emp_at_leastlp for eq.1/self_emp_both_p, self_emp_

only_m and self_emp_only_f for eq.2); (b) is (a) plusmale, no_sibling, one_sibling, two_siblings, income2, income3, income4, income5, income6, income 7, m_low, m_upper_sec, f_low, f_upper_sec, urban and severity; (c) is (b) plus dist050_2Iasi, dist50100_2Iasi and dist100150_2Iasi; (d) means (c) plusbac_avg_grade and high_school_avg_grade; (e) is (d) plus faith_god_both_p, faith_god_only_m, faith_god_only_f, secular and high_believe_god; (f) is (e) plus conscientiousness, agreeableness and openness; (g) is (f) plus interpers_trust2, interpers_trust3, interpers_trust 4 , interpers_trust5 and trust_no_instit, trust_governm, trust_church and trust_in_legal_instit; (h) is (g) plusmigr_index; (i) is (h) plusstate_welfare, state_must_inv_more_in_ec, compet_stim_effect and fear_of_compet; (j) is (i) plus labor_success_source and indiv_libert_progr_cause; $(\mathrm{k})$ is $(\mathrm{j})$ plus undesrv_fin_ advtg and avoid_buy_pub_transp_tickts, (1) is (k) plus child_needs_fam2dev and $p_{-}$

asum_resp4child_future - all abbreviations ofvariableswere explained in Table 1.

Note 3: Only those variables (lines) having a significant influence (minimum 10\%) at least for one set of specifications (column) were left in this table. 
Determinants of Entrepreneurial Proclivity of Students in Economics and Business Administration

Table 3 presents the MNL coefficients for estimating (eq.1) the probability to choose the outcome 2 (employee in a SME). The variable self_emp_at_leastlp is weak and not statistically significant. It remains insignificant along all our 12 successive scenarios.

Table 3. MNL coefficients (Ln of odds ratios) for estimating the probability to fit the professional category corresponding to outcome 2 - employee in a SME (eq.1)

\begin{tabular}{|c|c|c|c|c|c|c|c|c|c|c|c|c|}
\hline $\begin{array}{l}\text { Probability of outcome2 } \\
\text { (employee in a SME) }\end{array}$ & (a) & (b) & (c) & (d) & (e) & (f) & (g) & (h) & (i) & (j) & (k) & (1) \\
\hline male & & $\begin{array}{l}\mathbf{0 . 5 6 * *} \\
(0.25) \\
\end{array}$ & $\begin{array}{l}\mathbf{0 . 5 7 * *} \\
(0.25) \\
\end{array}$ & $\begin{array}{l}\mathbf{0 . 7 6 * * *} \\
(0.27)\end{array}$ & $\begin{array}{l}\mathbf{0 . 6 6 * *} \\
(0.27)\end{array}$ & $\begin{array}{l}\mathbf{0 . 6 * *} \\
(0.28)\end{array}$ & $\begin{array}{l}\mathbf{0 . 6 3 * *} \\
(0.29)\end{array}$ & $\begin{array}{l}\mathbf{0 . 6 4 * *} \\
(0.29)\end{array}$ & $\begin{array}{l}\mathbf{0 . 6 4 * *} \\
(0.29) \\
\end{array}$ & $\begin{array}{l}\mathbf{0 . 6 1 * *} \\
(0.29) \\
\end{array}$ & $\begin{array}{l}\mathbf{0 . 7 * *} \\
(0.30)\end{array}$ & $\begin{array}{l}\mathbf{0 . 7 * *} \\
(0.30)\end{array}$ \\
\hline no_sibling & & $\begin{array}{l}-0.23 \\
(0.42)\end{array}$ & $\begin{array}{l}-\mathbf{0 . 2 1} \\
(0.42)\end{array}$ & $\begin{array}{l}-0.5 \\
(0.45)\end{array}$ & $\begin{array}{l}-0.64 \\
(0.45)\end{array}$ & $\begin{array}{l}-0.68 \\
(0.45)\end{array}$ & $\begin{array}{l}-\mathbf{0 . 8 4 *} \\
(0.46)\end{array}$ & $\begin{array}{l}-0.85^{*} \\
(0.47)\end{array}$ & $\begin{array}{l}-\mathbf{0 . 8 5 *} \\
(0.47)\end{array}$ & $\begin{array}{l}-\mathbf{0 . 8 4 *} \\
(0.47)\end{array}$ & $\begin{array}{l}-0.9 * \\
(0.47)\end{array}$ & $\begin{array}{l}-0.9 * \\
(0.47)\end{array}$ \\
\hline bac_avg_grade & & & & $\begin{array}{l}\mathbf{0 . 3 4 * *} \\
(0.15)\end{array}$ & $\begin{array}{l}0.36^{* * *} \\
(0.15)\end{array}$ & $\begin{array}{l}\mathbf{0 . 3 6 * *} \\
(0.15)\end{array}$ & $\begin{array}{l}\text { 0.33*** } \\
(0.15)\end{array}$ & $\begin{array}{l}0.35^{* *} \\
(0.15)\end{array}$ & $\begin{array}{l}0.35^{* * *} \\
(0.15)\end{array}$ & $\begin{array}{l}\mathbf{0 . 3 4 * *} \\
(0.15)\end{array}$ & $\begin{array}{l}\mathbf{0 . 3 3 * *} \\
(0.15)\end{array}$ & $\begin{array}{l}\mathbf{0 . 3 3 ^ { * * * }} \\
(0.15)\end{array}$ \\
\hline high_school_avg_grade & & & & $\begin{array}{l}0.25 \\
(0.19)\end{array}$ & $\begin{array}{l}0.27 \\
(0.20)\end{array}$ & $\begin{array}{l}0.29 \\
(0.20)\end{array}$ & $\begin{array}{l}0.37 \\
(0.20)\end{array}$ & $\begin{array}{l}0.39 * \\
(0.20)\end{array}$ & $\begin{array}{l}0.39^{*} \\
(0.20)\end{array}$ & $\begin{array}{l}\mathbf{0 . 4 1}^{* * *} \\
(0.21)\end{array}$ & $\begin{array}{l}0.4^{* * *} \\
(0.21)\end{array}$ & $\begin{array}{l}\text { 0.41*** } \\
(0.21)\end{array}$ \\
\hline high_believe_god & & & & & $\begin{array}{l}-0.57^{*} \\
(0.31)\end{array}$ & $\begin{array}{l}-\mathbf{0 . 5 5 *} \\
(0.31) \\
\end{array}$ & $\begin{array}{l}-\mathbf{0 . 4 4} \\
(0.33)\end{array}$ & $\begin{array}{l}-\mathbf{0 . 4 1} \\
(0.33)\end{array}$ & $\begin{array}{l}-\mathbf{0 . 3 6} \\
(0.33)\end{array}$ & $\begin{array}{l}-\mathbf{- 0 . 3 8} \\
(0.33)\end{array}$ & $\begin{array}{l}-0.35 \\
(0.33)\end{array}$ & $\begin{array}{l}-0.36 \\
(0.33) \\
\end{array}$ \\
\hline conscientiousness & & & & & & $\begin{array}{l}-\mathbf{- 0 . 8 * *} \\
(0.40)\end{array}$ & $\begin{array}{l}\mathbf{- 0 . 8 6 * *} \\
(0.41)\end{array}$ & $\begin{array}{l}-\mathbf{- 0 . 8 2 * *} \\
(0.41)\end{array}$ & $\begin{array}{l}-\mathbf{- 0 . 7 5 *} \\
(0.41)\end{array}$ & $\begin{array}{l}\mathbf{- 0 . 6 7} \\
(0.42) \\
\end{array}$ & $\begin{array}{l}-\mathbf{0 . 6 7} \\
(0.42)\end{array}$ & $\begin{array}{l}\mathbf{- 0 . 6 7} \\
(0.42) \\
\end{array}$ \\
\hline trust_no_instit & & & & & & & $\begin{array}{l}\text { 108*** } \\
(0.42)\end{array}$ & $\begin{array}{l}\text { 109*** } \\
(0.42)\end{array}$ & $\begin{array}{l}\text { 1.1**** } \\
(0.43)\end{array}$ & $\begin{array}{l}\text { 1.1*k*k } \\
(0.43)\end{array}$ & $\begin{array}{l}\text { 1.07**** } \\
(0.43)\end{array}$ & $\begin{array}{l}\mathbf{1 . 0 7 * * * *} \\
(0.43)\end{array}$ \\
\hline trust_in_legal_instit & & & & & & & $\begin{array}{l}-\mathbf{0 . 4 7 *} \\
(0.26)\end{array}$ & $\begin{array}{l}-0.43^{*} \\
(0.26)\end{array}$ & $\begin{array}{l}-\mathbf{- 0 . 4 1} \\
(0.26)\end{array}$ & $\begin{array}{l}-\mathbf{- 0 . 3 7} \\
(0.26)\end{array}$ & $\begin{array}{l}-0.35 \\
(0.26)\end{array}$ & $\begin{array}{l}-\mathbf{0 . 3 4} \\
(0.26)\end{array}$ \\
\hline migr_index & & & & & & & & $\begin{array}{l}\mathbf{0}^{\mathbf{*}} \\
(0.06)\end{array}$ & $\begin{array}{l}\mathbf{0 . 1 1 ^ { * }} \\
(0.06)\end{array}$ & $\begin{array}{l}\mathbf{0 . 1}^{*} \\
(0.06)\end{array}$ & $\begin{array}{l}\mathbf{0}^{*} \\
(0.06)\end{array}$ & $\begin{array}{l}\mathbf{0}^{*} \mathbf{0}^{*} \\
(0.06)\end{array}$ \\
\hline compet_stim_effect & & & & & & & & & $\begin{array}{l}-0.73^{*} \\
(0.38) \\
\end{array}$ & $\begin{array}{l}-0.7 * \\
(0.38) \\
\end{array}$ & $\begin{array}{l}-0.7 * \\
(0.39)\end{array}$ & $\begin{array}{l}-0.7 * \\
(0.39)\end{array}$ \\
\hline Iabor_success_source & & & & & & & & & & $\begin{array}{l}-\mathbf{- 1 . 1 *} \\
(0.64) \\
\end{array}$ & $\begin{array}{l}\mathbf{- 1 . 1 2} * \\
(0.65)\end{array}$ & $\begin{array}{l}-\mathbf{- 1 . 1 2 *} \\
(0.65)\end{array}$ \\
\hline Observations & 1100 & 1100 & 1100 & 1038 & 1038 & 1038 & 1038 & 1038 & 1038 & 1038 & 1036 & 1036 \\
\hline $\begin{array}{l}\text { F-stat } \\
\text { [p-value] }\end{array}$ & $\begin{array}{l}18.74 \\
{[0.0003]}\end{array}$ & $\begin{array}{l}\mathbf{8 4 . 5 3} \\
{[0.0022]}\end{array}$ & $\begin{array}{l}\mathbf{9 3 . 5 3} \\
{[0.0036]}\end{array}$ & $\begin{array}{l}119.63 \\
{[0.0001]}\end{array}$ & $\begin{array}{l}\mathbf{1 3 8 . 3 9} \\
{[0.0001]}\end{array}$ & $\begin{array}{l}\mathbf{1 5 7 . 6 1} \\
{[0.0000]}\end{array}$ & $\begin{array}{l}195.05 \\
{[0.0000]}\end{array}$ & $\begin{array}{l}201.22 \\
{[0.0000]}\end{array}$ & $\begin{array}{l}219.47 \\
{[0.0000]}\end{array}$ & $\begin{array}{l}233.7 \\
{[0.0000]}\end{array}$ & $\begin{array}{l}246.29 \\
{[0.0000]}\end{array}$ & $\begin{array}{l}249.35 \\
{[0.0000]}\end{array}$ \\
\hline Pseudo R square & 0.007 & 0.031 & 0.035 & 0.048 & 0.057 & 0.065 & 0.083 & 0.086 & 0.095 & 0.103 & 0.11 & 0.111 \\
\hline
\end{tabular}

Source, Notes 1, 2 and 3 are the same as in Table 2.

After all these 12 scenarios, the variables no_sibling, compet_stim_effect and labour_success_source indicate a negative effect on the propesnsity towards SMEs. A positive effect towards choosing to work in a SME after graduation manifest the variables migr_index, trust_no_instit, high_school_avg_grade and bac_avg_grade. 
Aurelian-Petrus Plopeanu, Daniel Homocianu, Dinu Airinei

Table 4. MNL coefficients ( $\mathrm{Ln}$ of odds ratios) for estimating the probability to fit the professional category corresponding to outcome 3- employee in a LPC (eq.1).

\begin{tabular}{|c|c|c|c|c|c|c|c|c|c|c|c|c|}
\hline $\begin{array}{l}\text { Probability of outcome } 3 \\
\text { (employee in a LPC) }\end{array}$ & (a) & (b) & (c) & (d) & (e) & (f) & (g) & (h) & (i) & (j) & (k) & (1) \\
\hline self_emp_at_least1p & $\begin{array}{l}\mathbf{0 . 7 1 * * * *} \\
(0.24)\end{array}$ & 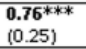 & $\begin{array}{l}\mathbf{0 . 7 4 * * * *} \\
(0.25)\end{array}$ & $\begin{array}{l}0.7 * * * \\
(0.26) \\
\end{array}$ & $\begin{array}{l}\text { 0.65**** } \\
(0.26)\end{array}$ & $\begin{array}{l}\mathbf{0 . 6 9 * * * *} \\
(0.28)\end{array}$ & $\begin{array}{l}0.7 * k * \\
(0.29)\end{array}$ & 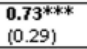 & $\begin{array}{l}\mathbf{0 . 7 6 ^ { * * * * }} \\
(0.29)\end{array}$ & 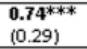 & $\begin{array}{l}\mathbf{0 . 7 * *} \\
(0.29)\end{array}$ & $\begin{array}{l}0.7 * * \\
(0.29)\end{array}$ \\
\hline male & & $\begin{array}{l}0.39 * \\
(0.21)\end{array}$ & $\begin{array}{l}\mathbf{0 . 4 1 * *} \\
(0.21)\end{array}$ & $\begin{array}{l}0.6^{* * * *} \\
(0.23)\end{array}$ & $\begin{array}{l}\mathbf{0 . 5 1 * * *} \\
(0.24)\end{array}$ & $\begin{array}{l}0.5^{* *} \\
(0.24)\end{array}$ & $\begin{array}{l}\mathbf{0 . 5 3 * *} \\
(0.25)\end{array}$ & $\begin{array}{l}0.53^{* * *} \\
(0.25)\end{array}$ & $\begin{array}{l}0.55^{* * *} \\
(0.26)\end{array}$ & $\begin{array}{l}\mathbf{0 . 5 2 * *} \\
(0.26)\end{array}$ & $\begin{array}{l}\mathbf{0 . 5 6 * *} \\
(0.26)\end{array}$ & $\begin{array}{l}0.55^{* * *} \\
(0.26)\end{array}$ \\
\hline no_sibling & & $\begin{array}{l}-0.46 \\
(0.35)\end{array}$ & $\begin{array}{l}-0.42 \\
(0.36)\end{array}$ & $\begin{array}{l}-0.64^{*} \\
(0.38)\end{array}$ & $\begin{array}{l}-0.77 * * \\
(0.39)\end{array}$ & $\begin{array}{l}-0.77^{* * *} \\
(0.39)\end{array}$ & $\begin{array}{l}-0.86^{* * *} \\
(0.40)\end{array}$ & $\begin{array}{l}-\mathbf{0 . 8 5 * *} \\
(0.40)\end{array}$ & $\begin{array}{l}-0.87^{* * *} \\
(0.40)\end{array}$ & $\begin{array}{l}-0.85 * * \\
(0.40)\end{array}$ & $\begin{array}{l}-0.91 * * \\
(0.40)\end{array}$ & $\begin{array}{l}-0.95 * * \\
(0.41)\end{array}$ \\
\hline one_sibling & & $\begin{array}{l}-0.35 \\
(0.32)\end{array}$ & $\begin{array}{l}-\mathbf{- 0 . 3} \\
(0.32)\end{array}$ & $\begin{array}{l}-\mathbf{0 . 5 1} \\
(0.35)\end{array}$ & $\begin{array}{l}-\mathbf{0 . 6 1 *} \\
(0.35) \\
\end{array}$ & $\begin{array}{l}-\mathbf{0 . 6 1 *} \\
(0.35) \\
\end{array}$ & $\begin{array}{l}-0.6^{*} \\
(0.36)\end{array}$ & $\begin{array}{l}-0.6^{*} \\
(0.36)\end{array}$ & $\begin{array}{l}-0.64 * \\
(0.36)\end{array}$ & $\begin{array}{l}-\mathbf{0 . 6 1 *} \\
(0.36) \\
\end{array}$ & $\begin{array}{l}-\mathbf{0 . 6 4 *} \\
(0.37)\end{array}$ & $\begin{array}{l}-0.67 * \\
(0.37) \\
\end{array}$ \\
\hline incomes & & $\begin{array}{l}-0.5 \\
(0.42)\end{array}$ & $\begin{array}{l}-\mathbf{- 0 . 4 3} \\
(0.42)\end{array}$ & $\begin{array}{l}-\mathbf{- 0 . 7} \\
(0.45)\end{array}$ & $\begin{array}{l}-\mathbf{- 0 . 7 4 ^ { * }} \\
(0.45)\end{array}$ & $\begin{array}{l}-0.76^{*} \\
(0.46)\end{array}$ & $\begin{array}{l}-0.65 \\
(0.47)\end{array}$ & $\begin{array}{l}-\mathbf{- 0 . 6 8} \\
(0.47)\end{array}$ & $\begin{array}{l}-\mathbf{0 . 6 9} \\
(0.47)\end{array}$ & $\begin{array}{l}-0.7 \\
(0.47)\end{array}$ & $\begin{array}{l}\mathbf{- 0 . 7 4} \\
(0.48)\end{array}$ & $\begin{array}{l}-0.75 \\
(0.48)\end{array}$ \\
\hline urban & & $\begin{array}{l}\mathbf{0 . 1 6} \\
(0.18)\end{array}$ & $\begin{array}{l}\mathbf{0 . 2 3} \\
(0.18)\end{array}$ & $\begin{array}{l}\mathbf{0 . 3 2 *} \\
(0.19)\end{array}$ & $\begin{array}{l}\mathbf{0 . 2 5} \\
(0.20)\end{array}$ & $\begin{array}{l}\mathbf{0 . 2 5} \\
(0.20)\end{array}$ & $\begin{array}{l}\mathbf{0 . 2 7} \\
(0.20)\end{array}$ & $\begin{array}{l}\mathbf{0 . 2 8} \\
(0.20)\end{array}$ & $\begin{array}{l}\mathbf{0 . 2 8} \\
(0.20)\end{array}$ & $\begin{array}{l}\mathbf{0 . 2 7} \\
(0.20)\end{array}$ & $\begin{array}{l}\mathbf{0 . 3 1} \\
(0.21)\end{array}$ & $\begin{array}{l}\mathbf{0 . 3} \\
(0.21)\end{array}$ \\
\hline dist050_2lasi & & & $\begin{array}{l}-0.53^{* * *} \\
(0.27)\end{array}$ & $\begin{array}{l}-0.57 * * \\
(0.29)\end{array}$ & $\begin{array}{l}-\mathbf{0 . 6 1 * *} \\
(0.29)\end{array}$ & $\begin{array}{l}-\mathbf{- 0 . 6 1 * * *} \\
(0.29)\end{array}$ & $\begin{array}{l}-\mathbf{- 0 . 6 9 * *} \\
(0.30)\end{array}$ & $\begin{array}{l}-\mathbf{- 0 . 6 6 * * k} \\
(0.30)\end{array}$ & $\begin{array}{l}-0.68^{* * *} \\
(0.30)\end{array}$ & $\begin{array}{l}-0.7 * * \\
(0.30)\end{array}$ & $\begin{array}{l}-\mathbf{0 . 7 3 * *} \\
(0.30)\end{array}$ & $\begin{array}{l}-0.73^{* * *} \\
(0.30) \\
\end{array}$ \\
\hline bac_avg_grade & & & & $\begin{array}{l}0.34^{\text {*k*k }} \\
(0.12)\end{array}$ & $\begin{array}{l}0.35 \text { *k*k } \\
(0.12)\end{array}$ & $\begin{array}{l}\mathbf{0 . 3 4 * k * k} \\
(0.12)\end{array}$ & 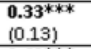 & $\begin{array}{l}\mathbf{0 . 3 5 * * *} \\
(0.13)\end{array}$ & $\begin{array}{l}\mathbf{0 . 3 6} \text { ***** } \\
(0.13)\end{array}$ & $\begin{array}{l}\mathbf{0 . 3 5 * * * *} \\
(0.13)\end{array}$ & $\begin{array}{l}\text { 0.35*k*k } \\
(0.13)\end{array}$ & $\begin{array}{l}\mathbf{0 . 3 5 * * *} \\
(0.13)\end{array}$ \\
\hline high_school_avg_grade & & & & $\begin{array}{l}\mathbf{0 . 3 7 * *} \\
(0.16)\end{array}$ & $\begin{array}{l}0.39^{* * k} \\
(0.17)\end{array}$ & $\begin{array}{l}0.39 * * \\
(0.17)\end{array}$ & $\begin{array}{l}0.43^{* * * * k} \\
(0.17)\end{array}$ & $\begin{array}{l}0.45^{* * * *} \\
(0.17)\end{array}$ & $\begin{array}{l}0.47 * * * * \\
(0.17)\end{array}$ & $\begin{array}{l}0.47 * * * * \\
(0.17)\end{array}$ & $\begin{array}{l}0.45 * k * k \\
(0.18)\end{array}$ & $\begin{array}{l}0.44^{* * * *} \\
(0.18)\end{array}$ \\
\hline faith_god_both_P & & & & & $\begin{array}{l}-\mathbf{- 0 . 5 2 *} \\
(0.30)\end{array}$ & $\begin{array}{l}-0.5^{*} \\
(0.31)\end{array}$ & $\begin{array}{l}-0.46 \\
(0.31)\end{array}$ & $\begin{array}{l}-0.46 \\
(0.31)\end{array}$ & $\begin{array}{l}-0.48 \\
(0.32)\end{array}$ & $\begin{array}{l}-0.49 \\
(0.32)\end{array}$ & $\begin{array}{l}-\mathbf{0 . 5 6 *} \\
(0.32)\end{array}$ & $\begin{array}{l}-\mathbf{0 . 5 8 *} \\
(0.33)\end{array}$ \\
\hline trust_in_legal_instit & & & & & & & $\begin{array}{l}-\mathbf{0 . 8 4 ^ { * * * * }} \\
(0.22) \\
\end{array}$ & $\begin{array}{l}-0.79^{* * * *} \\
(0.22)\end{array}$ & $\begin{array}{l}-0.79 * * * \\
(0.22) \\
\end{array}$ & $\begin{array}{l}-\mathbf{- 0 . 8 2 * * * *} \\
(0.22) \\
\end{array}$ & $\begin{array}{l}-0.8^{* 0 * * *} \\
(0.22) \\
\end{array}$ & $\begin{array}{l}-\mathbf{- 0 . 8 1 ^ { * * * * * }} \\
(0.22) \\
\end{array}$ \\
\hline migr_index & & & & & & & & 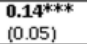 & $\begin{array}{l}\mathbf{0 . 1 5 * * * *} \\
(0.05)\end{array}$ & $\begin{array}{l}\mathbf{0 . 1 4 * * * * *} \\
(0.05)\end{array}$ & 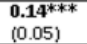 & $\begin{array}{l}\mathbf{0 . 1 4 * * *} \\
(0.05)\end{array}$ \\
\hline indiv_libert_progr_cause & & & & & & & & & & $\begin{array}{l}\mathbf{0 . 4 2 * *} \\
(0.21)\end{array}$ & $\begin{array}{l}\mathbf{0 . 4 2 * *} \\
(0.21)\end{array}$ & $\begin{array}{l}\mathbf{0 . 4 1 * *} \\
(0.21) \\
(0.21)\end{array}$ \\
\hline undesr_fin_advtg & & & & & & & & & & & $\begin{array}{l}-0.79 * * \\
(0.36) \\
\end{array}$ & $\begin{array}{l}-\mathbf{- 0 . 8 1 ^ { * * * }} \\
(0.36) \\
\end{array}$ \\
\hline Observations & 1100 & 1100 & 1100 & $\begin{array}{ll}1038 \\
\end{array}$ & 1038 & 1038 & 1038 & 1038 & 1038 & 1038 & 1036 & 1036 \\
\hline $\begin{array}{l}\text { F-stat } \\
\text { [p-value] }\end{array}$ & $\begin{array}{l}\mathbf{1 8 . 7 4} \\
{[0.0003]}\end{array}$ & $\begin{array}{l}84.53 \\
{[0.0022]}\end{array}$ & $\begin{array}{l}\mathbf{9 3 . 5 3} \\
{[0.0036]}\end{array}$ & $\begin{array}{l}19.63 \\
{[0.0001]}\end{array}$ & $\begin{array}{l}\mathbf{1 3 8 . 3 9} \\
{[0.0001]}\end{array}$ & $\begin{array}{l}\mathbf{1 5 7 . 6 1} \\
{[0.0000]}\end{array}$ & $\begin{array}{l}\mathbf{1 9 5 . 0 5} \\
{[0.0000]}\end{array}$ & $\begin{array}{l}\mathbf{2 0 1 . 2 2} \\
{[0.0000]}\end{array}$ & $\begin{array}{l}\mathbf{2 1 9 . 4 7} \\
{[0.0000]}\end{array}$ & $\begin{array}{l}233.7 \\
{[0.0000]}\end{array}$ & $\begin{array}{l}246.29 \\
{[0.0000]}\end{array}$ & $\begin{array}{l}249.35 \\
{[0.0000]}\end{array}$ \\
\hline Pseudo R square & 0.007 & 0.031 & 0.035 & 0.048 & 0.057 & 0.065 & 0.083 & 0.086 & 0.095 & 0.103 & 0.11 & 0.111 \\
\hline
\end{tabular}

Source, Notes 1, 2 and 3 are the same as in Table 2.

In the case of LPCs (Table 4), the influence of variable self_emp_at_leastlp is strong and significant. We also found a significant positive influence - scenario (b) of the masculine model (male variable) on the likelihood of students in economics and business administration to work in a LPC after graduation rather than in a PCI.

After all 12 sets of specifications, the negative influence of the variables no_sibling, dist050_2Iasi, faith_god_both_p, trust_in_legal_instit and undesrv_fin_

advtg remains in association with the decision to choose to work for a LPC. A positive one manifests bac_avg_grade, high_school_avg_grade, migr_index and indiv_libert_progr_cause. 
Determinants of Entrepreneurial Proclivity of Students in Economics and Business Administration

Table 5. MNL coefficients for estimating the probability of choosing first 3outcomes - focus on family entrepreneurial background (three core variables - eq.2).

\begin{tabular}{|c|c|c|c|c|c|c|c|c|c|c|c|c|}
\hline $\begin{array}{l}\text { Probability of } \\
\text { outcome1 (SE) }\end{array}$ & (a) & (b) & (c) & (d) & (e) & (f) & (g) & (h) & (i) & (j) & (k) & (I) \\
\hline P & $\begin{array}{l}\mathbf{0 . 9 2 *} \\
(0.51)\end{array}$ & $\begin{array}{l}\mathbf{0 . 9 *} \\
(0.52)\end{array}$ & $\begin{array}{l}\mathbf{0 . 8 7 *} \\
(0.52)\end{array}$ & $\begin{array}{l}\mathbf{0 . 8 7 *} \\
(0.52)\end{array}$ & $\begin{array}{l}\mathbf{0 . 8 8 *} \\
(0.53)\end{array}$ & $\begin{array}{l}\mathbf{0 . 7 4} \\
(0.54)\end{array}$ & $\begin{array}{l}\mathbf{0 . 6 1} \\
(0.55)\end{array}$ & $\begin{array}{l}\mathbf{0 . 6 6} \\
(0.55)\end{array}$ & $\begin{array}{l}\mathbf{0 . 6 8} \\
(0.56)\end{array}$ & $\begin{array}{l}\mathbf{0 . 6 6} \\
(0.56)\end{array}$ & $\begin{array}{l}\mathbf{0 . 5 7} \\
(0.57)\end{array}$ & $\begin{array}{l}\mathbf{0 . 5 6} \\
(0.57)\end{array}$ \\
\hline elf & $\begin{array}{l}0.6 \\
(0.50)\end{array}$ & $\begin{array}{l}\mathbf{0 . 6 1} \\
(0.51)\end{array}$ & $\begin{array}{l}0.56 \\
(0.51)\end{array}$ & $\begin{array}{l}0.57 \\
(0.52) \\
\end{array}$ & $\begin{array}{l}0.58 \\
(0.52)\end{array}$ & $\begin{array}{l}0.4 \\
(0.53)\end{array}$ & $\begin{array}{l}0.56 \\
(0.55)\end{array}$ & $\begin{array}{l}0.54 \\
(0.55)\end{array}$ & $\begin{array}{l}0.65 \\
(0.55)\end{array}$ & $\begin{array}{l}0.65 \\
(0.56)\end{array}$ & $\begin{array}{l}0.59 \\
(0.56)\end{array}$ & $\begin{array}{l}0.57 \\
(0.56)\end{array}$ \\
\hline Iy_f $f$ & $\begin{array}{l}.36^{* * * * *} \\
(0.36)\end{array}$ & $\begin{array}{l}1.48 * * * * \\
(0.37)\end{array}$ & $\begin{array}{l}1.48^{* * * * *} \\
(0.37)\end{array}$ & $\begin{array}{l}1.4 * * * * \\
(0.39)\end{array}$ & $\begin{array}{l}1.29 * * * * \\
(0.39)\end{array}$ & $\begin{array}{l}\text { 1.12**** } \\
(0.41)\end{array}$ & $\begin{array}{l}1.12^{* * * *} \\
(0.41)\end{array}$ & $\begin{array}{l}\text { 1.15**** } \\
(0.41)\end{array}$ & $\begin{array}{l}1.16 * * * * \\
(0.41) \\
\end{array}$ & $\begin{array}{l}\text { 1.12**** } \\
(0.42)\end{array}$ & $\begin{array}{l}\mathbf{1 0 9} \text { **** } \\
(0.42) \\
\end{array}$ & $\begin{array}{l}\mathbf{1 . 1 * * * *} \\
(0.42) \\
\end{array}$ \\
\hline $\begin{array}{l}\text { Probability of } \\
\text { outcome2 } \\
\text { (employee in a SME) }\end{array}$ & & & & & & & & & & & & \\
\hline self_e & $\begin{array}{l}\mathbf{0 . 6 4} \\
(0.53)\end{array}$ & $\begin{array}{l}\mathbf{0 . 6 8} \\
(0.53)\end{array}$ & $\begin{array}{l}\mathbf{0 . 6 7} \\
(0.53)\end{array}$ & $\begin{array}{l}\mathbf{0 . 6 7} \\
(0.54)\end{array}$ & $\begin{array}{l}\mathbf{0 . 6 9} \\
(0.55)\end{array}$ & $\begin{array}{l}\mathbf{0 . 8 2} \\
(0.56)\end{array}$ & $\begin{array}{l}\mathbf{0 . 6 6} \\
(0.57)\end{array}$ & $\begin{array}{l}\mathbf{0 . 7 2} \\
(0.57)\end{array}$ & $\begin{array}{l}\mathbf{0 . 6 8} \\
(0.58)\end{array}$ & $\begin{array}{l}\mathbf{0 . 7} \\
(0.58)\end{array}$ & $\begin{array}{l}\mathbf{0 . 6 7} \\
(0.58)\end{array}$ & $\begin{array}{l}\mathbf{0 . 6 7} \\
(0.58)\end{array}$ \\
\hline self_emp_only_m & $\begin{array}{l}\mathbf{0 . 5 8} \\
(0.50)\end{array}$ & $\begin{array}{l}\mathbf{0 . 6 2} \\
(0.50)\end{array}$ & $\begin{array}{l}\mathbf{0 . 5 9} \\
(0.51)\end{array}$ & $\begin{array}{l}\mathbf{0 . 6 2} \\
(0.51)\end{array}$ & $\begin{array}{l}\mathbf{0 . 6 5} \\
(0.52)\end{array}$ & $\begin{array}{l}0.72 \\
(0.53)\end{array}$ & $\begin{array}{l}\mathbf{0 . 9 *} \\
(0.55)\end{array}$ & $\begin{array}{l}\mathbf{0 . 8 9 *} \\
(0.55)\end{array}$ & $\begin{array}{l}\mathbf{0 . 9 1 *}^{*} \\
(0.55)\end{array}$ & $\begin{array}{l}\mathbf{0 . 9 3 *} \\
(0.55)\end{array}$ & $\begin{array}{l}\mathbf{0 . 8 6} \\
(0.56)\end{array}$ & $\begin{array}{l}\mathbf{0 . 8 8} \\
(0.56)\end{array}$ \\
\hline & $\begin{array}{l}\mathbf{0 . 1 3} \\
(0.44) \\
\end{array}$ & $\begin{array}{l}0.16 \\
(0.44) \\
\end{array}$ & $\begin{array}{l}\mathbf{0 . 1 7} \\
(0.44) \\
\end{array}$ & $\begin{array}{l}0.09 \\
(0.47) \\
\end{array}$ & $\begin{array}{l}\mathbf{0 . 0 2} \\
(0.47) \\
\end{array}$ & $\begin{array}{l}\mathbf{0 . 1 1} \\
(0.49) \\
\end{array}$ & $\begin{array}{l}\mathbf{0 . 0 7} \\
(0.49) \\
\end{array}$ & $\begin{array}{l}\mathbf{0 . 1} \\
(0.49) \\
\end{array}$ & $\begin{array}{l}\mathbf{0 . 1} \\
(0.50)\end{array}$ & $\begin{array}{l}\mathbf{0 . 1} \\
(0.50) \\
\end{array}$ & $\begin{array}{l}\mathbf{0 . 0 6} \\
(0.50) \\
\end{array}$ & $\begin{array}{l}\mathbf{0 . 0 7} \\
(0.50)\end{array}$ \\
\hline $\begin{array}{l}\text { Probability of } \\
\text { outcome } 3 \\
\text { (employee in a LPC) }\end{array}$ & & & & & & & & & & & & \\
\hline th_p & $\begin{array}{l}\mathbf{0 . 4 2} \\
(0.48)\end{array}$ & $\begin{array}{l}\mathbf{0 . 4 5} \\
(0.48) \\
\end{array}$ & $\begin{array}{l}\mathbf{0 . 4 1} \\
(0.48)\end{array}$ & $\begin{array}{l}0.25 \\
(0.51)\end{array}$ & $\begin{array}{l}\mathbf{0 . 2 5} \\
(0.51)\end{array}$ & $\begin{array}{l}\mathbf{0 . 2 9} \\
(0.52)\end{array}$ & $\begin{array}{l}\mathbf{0 . 1 8} \\
(0.53)\end{array}$ & $\begin{array}{l}\mathbf{0 . 2 6} \\
(0.53)\end{array}$ & $\begin{array}{l}\mathbf{0 . 2 6} \\
(0.54)\end{array}$ & $\begin{array}{l}\mathbf{0 . 2 1} \\
(0.54)\end{array}$ & $\begin{array}{l}\mathbf{0 . 1 5} \\
(0.54)\end{array}$ & $\begin{array}{l}\mathbf{0 . 1 6} \\
(0.54)\end{array}$ \\
\hline self_emp_only_m & $\begin{array}{l}\mathbf{0 . 3 7} \\
(0.45)\end{array}$ & $\begin{array}{l}\mathbf{0 . 4} \\
(0.45)\end{array}$ & $\begin{array}{l}\mathbf{0 . 3 3} \\
(0.45)\end{array}$ & $\begin{array}{l}\mathbf{0 . 3 6} \\
(0.46)\end{array}$ & $\begin{array}{l}\mathbf{0 . 3 6} \\
(0.47)\end{array}$ & $\begin{array}{l}\mathbf{0 . 3 8} \\
(0.47)\end{array}$ & $\begin{array}{l}\mathbf{0 . 5 1} \\
(0.49)\end{array}$ & $\begin{array}{l}\mathbf{0 . 4 9} \\
(0.49)\end{array}$ & $\begin{array}{l}\mathbf{0 . 5 4} \\
(0.49)\end{array}$ & $\begin{array}{l}0.55 \\
(0.50)\end{array}$ & $\begin{array}{l}\mathbf{0 . 4 9} \\
(0.50)\end{array}$ & $\begin{array}{l}\mathbf{0 . 4 9} \\
(0.50)\end{array}$ \\
\hline$-210=-6$ & $\begin{array}{l}0.98 * * * * \\
(0.34)\end{array}$ & $\begin{array}{l}\mathbf{1 0 6 * * * *} \\
(0.34)\end{array}$ & $\begin{array}{l}\mathbf{1 0 6 * * * *} \\
(0.35)\end{array}$ & $\begin{array}{l}\text { 1.05**** } \\
(0.36)\end{array}$ & $\begin{array}{l}0.97 * * * * \\
(0.37)\end{array}$ & $\begin{array}{l}\text { 101**** } \\
(0.38)\end{array}$ & $\begin{array}{l}0.99^{* * * *} \\
(0.39)\end{array}$ & $\begin{array}{l}\mathbf{1 0 3 * * * *} \\
(0.39)\end{array}$ & $\begin{array}{l}\text { 1.05*** } \\
(0.39)\end{array}$ & $\begin{array}{l}.02^{* * * *} \\
(0.39)\end{array}$ & $\begin{array}{l}\text { 1***; } \\
(0.39)\end{array}$ & $\begin{array}{l}\text { 1***; } \\
(0.39)\end{array}$ \\
\hline Observations & 1100 & 1100 & 1100 & 1038 & 1038 & 1038 & 1038 & 1038 & 1038 & 1038 & 1036 & 1036 \\
\hline F-st & 25.5 & 92. & 101.61 & 128.21 & 146.71 & 165 & 202.43 & 208.48 & 226.49 & 241.11 & 253.64 & \\
\hline o R square & {$[0.0024]$} & $\frac{[0.002}{0.035}$ & $\frac{[0.0032]}{\mathbf{0 . 0 3 8}}$ & {$[0.0001]$} & {$[0.0001]$} & {$[0.0000]$} & {$[0.0000]$} & $\frac{[0.0000]}{0.09}$ & {$[0.0000]$} & {$[0.0000]$} & {$[0.0000]$} & $\frac{[0.0000]}{0.115}$ \\
\hline
\end{tabular}

Source, Notes 1 and 2 are the same as in Table 2.Note 3: Only the variables / lines corresponding to the model's core (eq.2) were left in this table.

The probability of respondents' to become SE after graduation (the in-depth model, eq.2) is given by the coefficients and significance resulted from our 12 scenarios. According to Table 5, a student whose father is SE is more likely to become one after graduation - scenario (a) and scenario (1). In the case of a student with only the mother - SE, the individual's probability to become one is insignificant, recording the lowest coefficients. In the third case, a student in economics and business administration whose both parents are SE could also be one, but the coefficients are significant only in the first five sets of specifications, then they become insignificant and lower.

In the case of working in a SME, the probability of an individual to work there is low and insignificant if both parents / just the father are SE. In the case when only the mother is SE, they are more likely to be employees in a SME, but this probability is statistically significant only starting with the 7th scenario.

The probability of starting a career in a LPC after graduation is significant only in the case of "only father is SE" - scenario (a) and scenario (1).

In the generic model (eq.1, Tables 2, 3 and 4) the high performers in terms of average of grades of the baccalaureate exam are more inclined to be employee in a LPC or SME rather than choose an entrepreneurial career or work in PCIs after graduation. The high performers in terms of average of high-school grades are more likely to choose to be SE or work for a LPC rather than for a SME or PCI.

DOI: 10.24818/18423264/52.3.18.07 
Aurelian-Petrus Plopeanu, Daniel Homocianu, Dinu Airinei

Moreover, in terms of migration intentions, those more tempted to migrate, are more likely to work for a LPC, SME or become a SE (in this order) rather than work for a PCI.

Starting from Table 6 (eq.1 and 2), we can conclude that a male respondent is more likely to choose to be SE if at least one of his parents has an entrepreneurial profession. The same influence, but with less magnitude and significance, was observed in the case of female respondents.For a more comprehensive analysis of the influence of PRMs on their offspring decision to become entrepreneurs after graduation, we have observed (eq.2, Table 6) that only female respondents, willing to choose an entrepreneurial-type profession, inherit their father's SE type of career. In the same analysis, the father's influence on his son's decision to become entrepreneur is significant only for a simple scenario (b) and negligible when considering the most comprehensive one (1). For both male and female respondents, the mother's influence is insignificant in all scenarios.

Table 6. Respondents' gender and MNL coefficients for estimating the probability of choosing first 3 outcomes - focus on family entrepreneurial background (eq.1\&2)

\begin{tabular}{|c|c|c|c|c|c|c|c|c|c|}
\hline \multirow[b]{2}{*}{ eq.1 } & \multicolumn{4}{|c|}{ Respondent's gender } & \multirow[b]{2}{*}{ eq2. } & \multicolumn{4}{|c|}{ Respondent's gender } \\
\hline & $\begin{array}{l}\text { male } \\
\text { (b) }\end{array}$ & $\begin{array}{l}\text { male } \\
\text { (I) }\end{array}$ & $\begin{array}{l}\text { female } \\
\text { (b) }\end{array}$ & $\begin{array}{l}\text { female } \\
\text { (I) }\end{array}$ & & $\begin{array}{l}\text { male } \\
\text { (b) }\end{array}$ & $\begin{array}{l}\text { male } \\
\text { (I) }\end{array}$ & $\begin{array}{l}\text { female } \\
\text { (b) }\end{array}$ & $\begin{array}{l}\text { female } \\
\text { (I) }\end{array}$ \\
\hline $\begin{array}{l}\text { Probability of outcome } 1 \\
\text { (SE) }\end{array}$ & & & & & $\begin{array}{l}\text { Probability of outcome } 1 \\
\text { (SE) }\end{array}$ & & & & \\
\hline \multirow[t]{3}{*}{ self_emp_at_least $1 p$} & $\begin{array}{l}2.82 * * * \\
(1.06)\end{array}$ & $\begin{array}{l}3.60 * * \\
(1.47)\end{array}$ & $\begin{array}{l}1.08 * * * \\
(0.31)\end{array}$ & $\begin{array}{l}0.71 * * \\
(0.37)\end{array}$ & self_emp_both_p & $\begin{array}{l}\mathbf{1 5 . 9 4} \\
(1,125.32)\end{array}$ & $\begin{array}{l}\mathbf{1 9 . 1 8} \\
(1,359.64)\end{array}$ & $\begin{array}{l}\mathbf{0 . 3 4} \\
(0.63)\end{array}$ & $\begin{array}{l}-0.28 \\
(0.71)\end{array}$ \\
\hline & & & & & self_emp_only_m & $\begin{array}{l}\mathbf{1 5 . 2 3} \\
(1,162.07)\end{array}$ & $\begin{array}{l}\mathbf{1 6 . 3 3} \\
(1,528.38)\end{array}$ & $\begin{array}{l}\mathbf{0 . 4 7} \\
(0.59)\end{array}$ & $\begin{array}{l}\mathbf{0 . 3} \\
(0.66)\end{array}$ \\
\hline & & & & & self_emp_only_f & $\begin{array}{l}2.04 * \\
(1.10)\end{array}$ & $\begin{array}{l}\mathbf{1 . 7 4} \\
(1.45)\end{array}$ & $\begin{array}{l}1.57 * * * \\
(0.40)\end{array}$ & $\begin{array}{l}1.22 * * * \\
(0.47)\end{array}$ \\
\hline $\begin{array}{l}\text { Probability of outcome } 2 \\
\text { (employee in a SME) }\end{array}$ & & & & & $\begin{array}{l}\text { Probability of outcome } 2 \\
\text { (employee in a SME) }\end{array}$ & & & & \\
\hline \multirow[t]{3}{*}{ self_emp_at_least1p } & $\begin{array}{l}2.52^{* *} \\
(1.10)\end{array}$ & $\begin{array}{l}\text { 3.16** } \\
(1.51)\end{array}$ & $\begin{array}{l}\mathbf{0 . 1 7} \\
(0.33)\end{array}$ & $\begin{array}{l}\mathbf{0 . 3} \\
(0.39)\end{array}$ & self_emp_both_p & $\begin{array}{l}\mathbf{1 5 . 1 2} \\
(1,125.33)\end{array}$ & $\begin{array}{l}\mathbf{1 7 . 6 1} \\
(1,359.64)\end{array}$ & $\begin{array}{l}\mathbf{0 . 4 6} \\
(0.56)\end{array}$ & $\begin{array}{l}\mathbf{0 . 5 9} \\
(0.62)\end{array}$ \\
\hline & & & & & self_emp_only_m & $\begin{array}{l}\mathbf{1 6} \\
(1,162.07)\end{array}$ & $\begin{array}{l}\mathbf{1 7 . 0 2} \\
(1,528.38)\end{array}$ & $\begin{array}{l}\mathbf{0 . 1} \\
(0.58)\end{array}$ & $\begin{array}{l}\mathbf{0 . 5 2} \\
(0.66)\end{array}$ \\
\hline & & & & & self_emp_only_f & $\begin{array}{l}1.22 \\
(1.22)\end{array}$ & $\begin{array}{l}\mathbf{1 . 0 8} \\
(1.57)\end{array}$ & $\begin{array}{l}-0.01 \\
(0.50)\end{array}$ & $\begin{array}{l}-0.06 \\
(0.57)\end{array}$ \\
\hline $\begin{array}{l}\text { Probability of outcome } 3 \\
\text { (employee in a LPC) }\end{array}$ & & & & & $\begin{array}{l}\text { Probability of outcome } 3 \\
\text { (employee in a LPC) }\end{array}$ & & & & \\
\hline \multirow[t]{3}{*}{ self_emp_at_least $1 p$} & $\begin{array}{l}2.6 * * * \\
(1.06)\end{array}$ & $\begin{array}{l}\mathbf{3 . 4 2 * *} \\
(1.45)\end{array}$ & $\begin{array}{l}\mathbf{0 . 5 9 * *} \\
(0.27)\end{array}$ & $\begin{array}{l}\mathbf{0 . 5 7 *} \\
(0.32)\end{array}$ & self_emp_both_p & $\begin{array}{l}\mathbf{1 5 . 2 2} \\
(1,125.33)\end{array}$ & $\begin{array}{l}\mathbf{1 8 . 0 5} \\
(1,359.64)\end{array}$ & $\begin{array}{l}\mathbf{0 . 1 4} \\
(0.51)\end{array}$ & $\begin{array}{l}-0.21 \\
(0.61)\end{array}$ \\
\hline & & & & & self_emp_only_m & $\begin{array}{l}\mathbf{1 5 . 3 6} \\
(1,162.07)\end{array}$ & $\begin{array}{l}\mathbf{1 6 . 2 4} \\
(1,528.38)\end{array}$ & $\begin{array}{l}\mathbf{0 . 1 8} \\
(0.48)\end{array}$ & $\begin{array}{l}\mathbf{0 . 4 1} \\
(0.54)\end{array}$ \\
\hline & & & & & self_emp_only_f & $\begin{array}{l}\mathbf{1 . 9 *} \\
(1.09)\end{array}$ & $\begin{array}{l}\mathbf{2 . 1 5} \\
(1.41)\end{array}$ & $\begin{array}{l}0.96 * * * \\
(0.37)\end{array}$ & $\begin{array}{l}\mathbf{0 . 9 3 * *} \\
(0.43)\end{array}$ \\
\hline Observations & 315 & 296 & 785 & 740 & Observations & 315 & 296 & 785 & 740 \\
\hline Pseudo R square & 0.073 & 0.272 & 0.03 & 0.126 & Pseudo R square & 0.08 & 0.284 & 0.035 & 0.132 \\
\hline
\end{tabular}

Source, Notes 1 and 2 are the same as in Table 2.Note 3: Only the variables / lines corresponding to the model's core (eq.1 / eq.2) were left in this table.

Similarly, we have tried to analyze the relation between the lack or the presence of openness and the effect of the core variables corresponding to PRMs on the respondent's' decision to become SE after graduation for both eq. 1 and 2 using the second (b) and the last set of specifications (l). Therefore, we confirm (Simone Chlosta et al. 2012, 128) a heavy influence making the core variables not 


\section{Determinants of Entrepreneurial Proclivity of Students in Economics and Business Administration}

to matter in terms of influence on increasing the likelihood that individuals become SE when openness as personality trait is missing (value 0 ).

\section{Testing the models}

The estimates of the "Area Under Curve" (AUC) for "Receiver Operating Characteristic curve" (AUROC) as a diagnostic test for our MNL model (eq.2, Table 5) by using Peterson's shared code (Leif E. Peterson 2010) in Stata indicate a value of $\sim 0.84$ (Fig.1).This means a good accuracy for the final and most comprehensive set of specifications(l), while for eq. 1 and the second set of specifications (b), the diagnostic test above returned an expected lower value of almost 0.5 (fail).

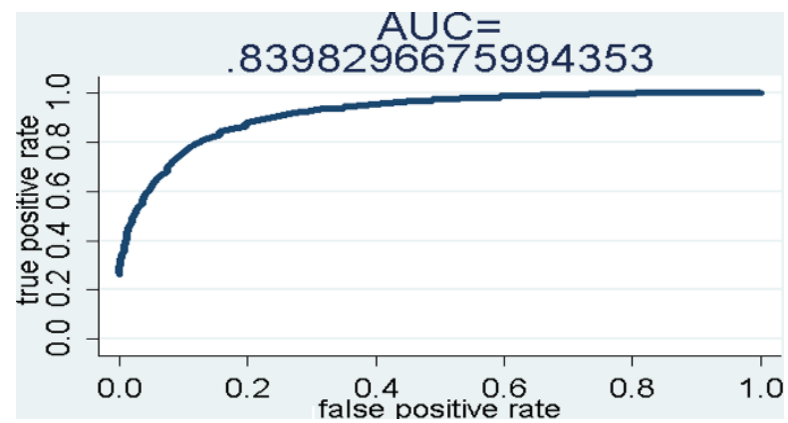

Figure 1. AUROC test results considering eq.2 and the final set of specifications (l)

The most well-known formal tests for testing the IIA (Independence of Irrelevant Alternatives) assumption are: Hausman-McFadden (1984), suest-based Hausman for safer results in case of negative values and Small-Hsiao (1985). The last one is less reliable because it randomly splits the overall sample into two subsamples modulating a restricted model and an unrestricted one. Thus, the random choice of subsamples generates different results for this test (Long and Freese 2006).

For all scenarios and for both eq. 1 and 2, the Hausman-McFadden and suestbased Hausman tests do not violate the IIA assumption. Our results for eq. 2 and the most comprehensive scenario, (1), using this test clearly show that the IIA assumption, on which the MNL model relies, was not rejected.

The usefulness of this kind of test in assessing the violations of IIA assumption has been considered insufficient, especially when the sample size is small and, therefore, doubted (Cheng and Long 2007). Hence, in order to overcome possible inconsistencies and limitations, we also tested the results using the multinomial probit (MNP) model and they proved to be very similar in terms of robustness when compared with those of the MNL model (both eq.1 and 2). Choosing this alternative to the MNL model is commonly discussed in the scientific literature (Train 2003).

DOI: 10.24818/18423264/52.3.18.07 
Aurelian-Petrus Plopeanu, Daniel Homocianu, Dinu Airinei

In order to conclude about the acceptance of IIA assumption, several authors (Cheng and Long 2007) consider that this assumption is mostly rejected when the alternatives are close substitutes (not the case of these four professional categories).

\section{Discussions on the findings and limitations of the study}

After we have included many relevant control variables to our model along those twelve scenarios from (a) to (1), the results prove to be robust to our sample composition. We have used the MNL model with three outcomes for the predicted categorical variable (SE as 1, employee in a SME as 2 or in a LPC as 3), constantly compared with the base outcome as 4, employee in a PCI, to test the effect of the independent variables considered above (see Tables 1-5).

We have decided to make the transition from a more generalist PRM ("at least one parent SE") to a more in-depth one, particularly trying to emphasize the paternal / maternal determinants (self_emp_only_m / self_emp_only_f / self_emp_both_p) of the propensity of a student to choose a certain career after graduation. In addition, this in-depth approach has brought other significant influences: self_emp_only_f $(1$ vs. 2 and, in addition, 3 vs. 2$)$, one_sibling (4 vs. 2 in addition), urban (new influence) with 3 vs. 2, dist050_2Iasi (2 vs. 3 in addition) and agreeableness (new one) with 3 vs. 1. Although with slight differences in values (coefficient and $p$ value), the rest of significant $(p<0.1)$ influences, common to both approaches, determine the same order by magnitude and significance for those four professional categories.

Initially, we included in the in-depth model only those three variables above (paternal and/or maternal determinants - eq.2). In the next steps, we included the same categories of variables as in the previous MNL regressions (Tables 2-4), by using the same sets of specifications from scenario (a) to (1). These new included scenarios improved in comparison with the initial one, explaining more and better, as shown by the value of pseudo- $\mathrm{R}^{2}$ from the last scenario, (l) - see Table 5 .

After we ran all the scenarios from (b) to (l), the estimated effect of the core (a) variables (eq.1 - self_emp_at_leastlp, eq.2 - only the 3rd one, namely self_emp_only_f), especially for two outcomes, namely SE and employee in a LPC, has slightly changed in magnitude, while the level of significance proved to be the same, emphasizing that the effect is robust across all specifications for both eq. 1 and 2.The respondents who have only theirfathers with an entrepreneurial-nature jobs (paternal role models of the entrepreneurial behavior) are more likely to dedicate their future careers to work as SE or for LPCs in the detriment of SMEs. Similarly, the male respondents are more likely to work as SE, then for a LPC. Moreover, the students whose mothers have upper secondary education are more likely to work for SMEs rather than act as SE or as employees for LPCs. This result complements some ideas emphasizing that successful entrepreneurs have higher-educated mothers, while the failed ones - viceversa.

In addition, the respondents with an increased level of parental severity are more likely to prefer to work as SE rather than to be a LPC employee (Fig.2). 


\section{Determinants of Entrepreneurial Proclivity of Students in Economics and Business Administration}

Besides, the students with a declared location in proximity of the city of Iasi (less than $50 \mathrm{~km}$ ) are more likely to follow a career in a SME rather than to work for a LPC. If belonging to an urban location they choose LPCs rather than SMEs (Fig.2).

In terms of school performance, increasing the average of baccalaureate grades translates in choosing to work for LPCs or SMEs rather than be a SE (Fig.2).

Moreover, in terms of personality traits, the students who manifest more conscientiousness seem more determined to prefer LPCs in the detriment of SMEs. When considering openness as personality trait, the results are interesting. Therefore, the respondents who manifest it are also more willing to choose their future career as SE rather than working for LPCs or SMEs. Regarding the agreeableness, those better defined by this feature tend to work for LPCs rather than act as SE after graduation (Fig.2). Considering the fact that the obedience is a component of agreeableness, therefore we can consider that those students who are willing to be SE after graduation are mannered and tolerant people by the frequency of responses.

Other strong negative influences towards the entrepreneurial proclivity are generated by the fear of competition and the view that the state must involve more in the economy. The students who consider that the state must involve more in the economy are more likely to work for LPCs rather than becoming SE. The last ones are inimical to any interference of the state in the economy and, consequently, they seem to be very liberal. Moreover, the students who emphasize a negative motivation for competition, namely the fear to compete for a job, are also more likely to start a career in a SME or in a LPC in the detriment of choosing to be SE after graduation (Fig.2). Therefore, those who want to run their own businesses are not afraid to compete with others for a desired outcome.The respondents suggesting no trust in institutions are more likely to work for SMEs rather than for larger ones or becoming SE. The students choosing to trust legal institutions are also more tempted to work for a SME rather than for a LPC after graduation (Fig.2).

Another interesting idea resulting from this paper is about the strong beliefs of the future SE individuals when questioned about their attitude towards the true value of labor. Therefore, it is worth noting that they do not appreciate at all the idea that labor is the source of success in life or in society. This striking finding defies the importance of labor in achieving desired goals in the long run, emphasized by several researches in this scientific field, as mentioned in the literature review section of this paper. Hence, the individuals who consider labor as a key factor of success in life and society are more likely to choose to work for LPCs rather than become SE.

Four other dichotomous, less decisive variables, have been identified when choosing to become SE. They suggest that those who agree that the individual 
Aurelian-Petrus Plopeanu, Daniel Homocianu, Dinu Airinei

freedom is a source of progress in life and society are more likely to choose to become SE or to work for LPCs rather than for SMEs (Fig.2).

A powerful influence of an apparently ethical judgment towards the entrepreneurial proclivity was also found: the respondents who choose to be SE after graduation also consider justified to avoid buying tickets for the public transport. Moreover, they are more determined to choose to be SE after graduation in the detriment of starting a career in a SME or in a LPC. This attitude should be associated with a certain tradition of avoiding to pay fees in this particular geopolitical area.

\begin{tabular}{|c|c|c|c|c|c|c|c|c|c|c|}
\hline Variable & utcome vs. Outcome & b 5 & SCORE $+=e^{\wedge}(b$ & WSTDx) & Variable & tcome vs. Outcome & b & $\mathrm{e}^{\lambda} \mathrm{b}$ & SCORE $=\mathrm{e}^{\lambda}\left(\mathrm{b}^{*}\right.$ & F(TDX) \\
\hline self_emp_only_f & $1 \times 5.4$ & 1.1030 .0093 .014 & 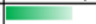 & 1.409 & self_emp_only_f & $2 \times 5.3$ & -0.9170 .018 & 0.400 & & 0.752 \\
\hline self_emp_only_f & 1 vs. 2 & 1.0300 .0142 .800 & $=$ & 1.377 & self_emp_only_f & 4 vs. 3 & -0.9910 .01 & 0.371 & 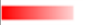 & 0.735 \\
\hline self_emp_only_f & 3 vs. 4 & 0.9910 .0112 .693 & $=$ & 1.360 & self_emp_only_f & 2 vs. 1 & -1.0300 .014 & 0.357 & 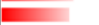 & 0.726 \\
\hline self_emp_only_f & 3 vs. 2 & 0.9170 .0182 .502 & $=$ & 1.330 & self_emp_only_f & 4 vs. 1 & -1.1030 .009 & 0.332 & 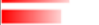 & 0.710 \\
\hline male & 185.4 & 1.0550 .0002 .871 & & 1.611 & male & $3 v 5.1$ & -0.4880 .030 & 0.614 & & 0.802 \\
\hline male & 2 vs. 4 & 0.6950 .0212 .003 & 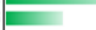 & 1.369 & male & 4 vs. 3 & -0.5670 .031 & 0.567 & 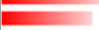 & 0.774 \\
\hline male & $3 v 5.4$ & 0.5670 .0311 .763 & 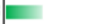 & 1.292 & male & 4 vs. 2 & -0.6950 .021 & 0.499 & 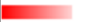 & 0.731 \\
\hline male & $1 \mathrm{vs.} 3$ & 0.4880 .0301 .629 & E & 1.247 & male & 4 vs. 1 & -1.0550 .000 & 0.348 & & 0.621 \\
\hline no_sibling & 4 vs.1 & 1.2560 .0083 .511 & & 1.665 & no_sibling & 3 vs. 4 & -0.9360 .022 & 0.392 & & 0.684 \\
\hline no_sibling & 4 vs. 2 & 0.9600 .0442 .612 & 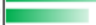 & 1.476 & no_sibling & 2 vs. 4 & -0.9600 .044 & 0.383 & $=$ & 0.677 \\
\hline no_sibling & 4 vs. 3 & 0.9360 .0222 .550 & 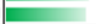 & 1.462 & no_sibling & 1 vs. 4 & -1.2560 .008 & 0.285 & & 0.601 \\
\hline one_sibling & $4 v 5.2$ & 0.7240 .0932 .063 & 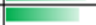 & 1.436 & one_sibling & 3 vs. 4 & -0.6620 .074 & 0.516 & 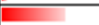 & 0.718 \\
\hline one_sibling & 4 vs. 3 & 0.6620 .0741 .938 & 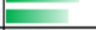 & 1.392 & one_sibling & 2 vs. 4 & -0.7240 .093 & 0.485 & 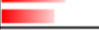 & 0.696 \\
\hline m_upper_sec & 2 vs.1 & 0.7670 .0312 .154 & 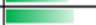 & 1.413 & m_upper_sec & $3 \mathrm{vs} .2$ & -0.6420 .043 & 0.526 & 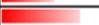 & 0.749 \\
\hline m_upper_sec & 2 vs. 3 & 0.6420 .0431 .900 & 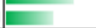 & 1.336 & m_upper_sec & 1 vs. 2 & -0.7670 .031 & 0.464 & 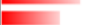 & 0.708 \\
\hline urban & $3 \mathrm{vs} .2$ & 0.3400 .0971 .405 & & 1.183 & urban & $2 \times 5.3$ & -0.3400 .097 & 0.712 & & 0.845 \\
\hline \begin{tabular}{|l} 
severity \\
\end{tabular} & $1 \mathrm{Vs} .3$ & 0.0680 .0881 .071 & & 1.172 & severity & $3 \times 5.1$ & -0.0680 .088 & 0.934 & E & 0.853 \\
\hline distriso__lasi & 4 vs.3 & 0.7490 .0142 .115 & & 1.430 & distro50_2lasi & $3 v 5.2$ & -0.5190 .087 & 0.595 & & 0.781 \\
\hline dist050_2lasi & 2 vs. 3 & 0.5190 .0871 .679 & $=$ & 1.281 & dist050_2lasi & 3 vs. 4 & -0.7490 .014 & 0.473 & 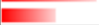 & 0.699 \\
\hline bac_avg_grade & $3 v 5.4$ & 0.3550 .0061 .426 & 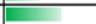 & 1.365 & bac_avg_grade & $1 \mathrm{vs} .3$ & -0.2250 .076 & 0.798 & & 0.821 \\
\hline bac_avg_grade & 2 vs. 4 & $0.3290 .033 \quad 1.389$ & $=$ & 1.334 & bac_avg_grade & 4 vs. 2 & & 0.720 & 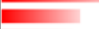 & \\
\hline bac_avg_grade & 3 vs.1 & $0.225 \quad 0.076 \quad 1.252$ & E & 1.218 & bac_avg_grade & 4 vs. 3 & -0.3550 .006 & 0.701 & 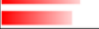 & 0.732 \\
\hline high_school_avg_grade & 1 1v5.4 & 0.4930 .0171 .637 & 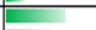 & 1.382 & high_school_avg_grade & $4 v 5.2$ & -0.4210 .044 & 0.657 & 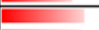 & 0.758 \\
\hline high_school_avg_grade & 3 vs. 4 & 0.4310 .0151 .538 & $=$ & 1.327 & high_school_avg_grade & 4 vs. 3 & -0.4310 .015 & 0.650 & 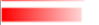 & 0.753 \\
\hline high_school_avg_grade & 2 vs. 4 & 0.4210 .0441 .523 & $=$ & 1.319 & high_school_avg grade & 4 vs. 1 & -0.4930 .017 & 0.611 & 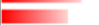 & 0.723 \\
\hline faith_god_both_p & 4 v5. 3 & 0.5920 .0691 .808 & 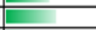 & 1.345 & fraith_god_both_p & $3 v 5.4$ & -0.5920 .069 & 0.553 & & 0.744 \\
\hline conscientiousness & $3 \times 5.2$ & 0.8450 .0122 .327 & 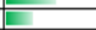 & 1.251 & conscientiousness & $2 v 5.3$ & -0.8450 .012 & 0.430 & 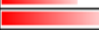 & 0.799 \\
\hline agreeableness & $3 \mathrm{VS.1}$ & 0.5970 .0991 .817 & & 1.146 & agreeableness & $1 \mathrm{vs} .3$ & -0.5970 .099 & 0.550 & & 0.873 \\
\hline openness & $1 \mathrm{vs} .3$ & 0.5980 .0061 .818 & 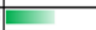 & 1.341 & openness & 4 vs. 1 & -0.4790 .063 & 0.619 & 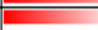 & 0.791 \\
\hline openness & 1 vs. 2 & 0.5470 .0321 .729 & $=$ & 1.308 & openness & 2 vs. 1 & -0.5470 .032 & 0.578 & 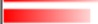 & 0.765 \\
\hline openness & 1 vs. 4 & 0.4790 .0631 .614 & 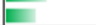 & 1.265 & openness & 3 vs. 1 & -0.5980 .006 & 0.550 & 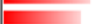 & 0.746 \\
\hline trust_no_instit & $2 \times 5.4$ & 1.0880 .0122 .969 & & 1.699 & trust_no_instit & 1 vs. 2 & -0.6960 .073 & 0.498 & 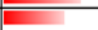 & 0.712 \\
\hline trust_no_instit & 2 vs. 3 & 0.9440 .0082 .570 & & 1.583 & trust_no_instit & 3 vs. 2 & -0.9440 .008 & 0.389 & & 0.632 \\
\hline trust_no_instit & 2 vs. 1 & 0.6960 .0732 .006 & 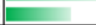 & 1.404 & trust_no_instit & 4 vs. 2 & -1.0880 .012 & 0.337 & & 0.589 \\
\hline trust_in_legal_instit & $4 \times 5.3$ & 0.8040 .0002 .233 & + & 1.399 & trust_in_legal_instit & $3 \times 5.2$ & -0.4340 .072 & 0.648 & & 0.834 \\
\hline trust_in_legal_instit & 4 vs. 1 & 0.7960 .0042 .217 & 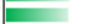 & 1.394 & trust_in_legal_instit & 1 vs. 4 & -0.7960 .004 & 0.451 & 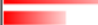 & 0.717 \\
\hline trust_in_legal_instit & 2 vs. 3 & 0.4340 .0721 .543 & E & 1.199 & trust_in_legal_instit & $3 \mathrm{vs}$ & -0.8040 .000 & 0.448 & 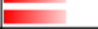 & 0.715 \\
\hline migr_index & $3 \times 5.4$ & 0.1410 .0051 .151 & 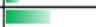 & 1.323 & migrindex & 4 v5.2 & -0.1040 .077 & 0.901 & & 0.813 \\
\hline migr_index & $2 v 5.4$ & 0.1040 .0771 .110 & E & 1.230 & migr_index & 4 vs. 3 & 10.005 & 0.869 & 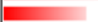 & 0.756 \\
\hline state_must_inv_more_in_ec & $3 \times 5.1$ & 0.5180 .0121 .679 & 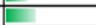 & 1.264 & state_must_inv_more_in_ec & $61 \mathrm{Vs} .3$ & -0.5180 .012 & 0.596 & 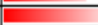 & \\
\hline Compet_stim_effect & $4 \times 5.2$ & 0.7080 .0682 .029 & 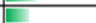 & 1.239 & compet_stim_effect & 2 v5. 4 & -0.7080 .068 & 0.493 & & 0.807 \\
\hline fear_of_compet & 2 v5.1 & 0.6420 .0051 .900 & 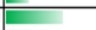 & 1374 & fear_of_compet & $1 \mathrm{vs}$ & & 0.691 & $=$ & \\
\hline fear_of_compet & 1 & 0.0161 .745 & $=$ & 1.317 & compet & 1 vs. 4 & -0.5570 & & 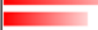 & 0.7 \\
\hline fear_of_compet & 3 vs. 1 & 0.3700 .0531 .447 & & 1.201 & fear_of_compet & 1 vs. 2 & -0.6420 .005 & 50.526 & 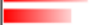 & 0.728 \\
\hline Tabor_success_source & $4 \times 5.1$ & 1.6180 .0095 .042 & 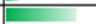 & 1.424 & abor_success_source & $2 v 5.4$ & -10990.090 & 50.333 & \begin{tabular}{|l|llll} 
\\
\end{tabular} & 0.787 \\
\hline labor_success_source & 3 vs. 1 & 1.1850 .0023 .269 & $=$ & 1.295 & labor_success_source & 1 vs. 3 & -1.1850 .002 & 20.306 & 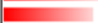 & 0.772 \\
\hline labor_success_source & 4 vs. 2 & 1.0990 .0903 .001 & $=$ & 1.271 & labor- & $1 \mathrm{v}$ & -1.6180 & 0.198 & 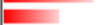 & 0.702 \\
\hline indiv_libert_progr_cause & $3 \times 5,2$ & 71.632 & - & 1.2. & indiv_libert_progr_cause & 4 vs. 3 & 50 & 0.657 & & 0.828 \\
\hline indiv_libert_progr_cause & 1 vs. 2 & 0.4290 .0791 .536 & & 1.213 & lindiv_libert_progr_cause & 2 vs. 1 & -0.4290 .079 & 0.651 & & 0.8 \\
\hline indiv_libert_progr_cause & 3 vs. 4 & $0.420 \quad 0.044 \quad 1.522$ & $E$ & 1.208 & indiv_liber_progr_cause & 2 vs. 3 & $-0.490 \quad 0.017$ & 0.613 & 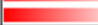 & 0.802 \\
\hline undesn_fin_advtg & 4 vs. 3 & 0.8140 .0252 .257 & 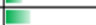 & 1.240 & undesry_fin_advtg & $3 \times 5.4$ & -0.8140 .025 & 50.443 & 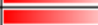 & 0.807 \\
\hline avoid_buy_pub_tran & $1 \times 5.2$ & & & 1.552 & avoid_buy_pub_trar & $3 \mathrm{vs}$ & & & & \\
\hline _ou & $s_{5} 1 \mathrm{vs}$ & 2.817 & $=$ & 1.347 & avoid_buy_pub_tra & & -1.0100 & & $=$ & \\
\hline avoid_buy_put_transp_tickts & $\begin{array}{l}5 \\
\text { v }\end{array}$ & 1.0100 .0172 .746 & $=$ & 1.337 & avoid_buy_pub_transp_tickts & ts 4 vs.1 & -1.0360 .011 & 0.355 & 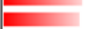 & 0.743 \\
\hline avoid_buy_pub_transp_tickts & 51 vs. 3 & 0.5200 .0691 .682 & & 1.161 & avoid_buy_pub_transp_tickts & ts 2 vs.1 & -1.5300 .001 & 0.216 & & 0.644 \\
\hline$\overline{\text { TOTA }}$ & & & & 73.763 & & & & & & \\
\hline
\end{tabular}

Figure 2. Ranking outcomes for all input variables starting from the entire list of significant $(\mathbf{p}<0.1)$ positive / negative coefficients (Stata - listcoef command) considering eq. 2 and the final set of specifications (l)

Studying the overall tendencies and reported results, we came also to the conclusion that in the perception of the students from North and NE part of 


\section{Determinants of Entrepreneurial Proclivity of Students in Economics and Business Administration}

Romania, the idea of working for a LPC means much more in terms of entrepreneurship foundation, spirit, involvement and effort than working for a SME.

A notable resemblance between outcome 1 and 3 (Fig.2) is mainly suggested by common positive influences such as: the PRMs, male respondent type, average of high-school grades and the attitude towards avoiding to buy public transport tickets. More, in terms of entrepreneurial features, the third outcome shows other specific attributes that are not common for the SE status, such as: conscientiousness and a positive consideration about the value of labor as a source of progress.

The most important limitation of this study relates to our survey design which offers a static picture at the moment of collecting data about the proclivity of students in economics and business administration to become SE after graduation. We cannot provide any final conclusion on their decision to become SE or not in the near future. Those who didn't want that at the moment of collecting the data could change their perspectives in the future.

Another limitations might be due to: the lack of a qualitative study amongst these students to better assess their subjective entrepreneurial decisions; the difficulty to check whether these students' parents are indeed entrepreneurs or SE, which we consider to be a minor issue mostly because the individuals who interpret the professional status of their parents as being entrepreneurs are determined to embrace the entrepreneurial character of their parent's activities (Simone Chlosta et al. 2012, 128); the missing opportunity to validate the initial hypotheses in other Romanian university centers which we will overcome by considering them in a further research, while our sample is still representative in the context of Eastern and NE Romanian regions' specific peculiarities we want to get acquainted with.

\section{Conclusions}

Our study demonstrates that even from a more general approach, when we consider at least the influence of one of the parents, the PRMs matter in terms of entrepreneurial proclivity, especially when we emphasize this influence on the male respondents, while less magnitude and significance are observed for the female students. More, we took into consideration a more in-depth approach of the PRMs by simultaneously taking into account three components: only the influence of the father (paternal role models), only of the mother (maternal role models) and both (PRM). The paternal role models proved to be important determinants for students in economics and business administration to become self-employed after graduation, especially when considering the intergenerational transfer of the entrepreneurial pattern from father to daughter.

The strong influence of the gender towards the students' proclivity to be SE after graduation, in our case positive for the male respondents, is another interesting finding. This paper also underlines that openness, one of the most important personality traits of an individual, is a key feature with positive influence

DOI: 10.24818/18423264/52.3.18.07 
Aurelian-Petrus Plopeanu, Daniel Homocianu, Dinu Airinei

for those students who are inclined to choose to be SE after graduation. The results from our paper also show that openness conditions the influence of PRMs and this finding is in line with other papers relating with this topic, as shown at the end of section No.4.

\section{REFERENCES}

[1] Ács, Z., Szerb, L. (2009), The Global Entrepreneurship Index (GEINDEX). Foundations and Trends ${ }^{\circledR}$ in Entrepreneurship, 5(5), 341-435;

[2] Brixy, U., Sternberg, R., Vorderwlbecke, A. (2013), Business Start-ups by Migrants. IAB Brief Report 25. Nurnberg: Institute for Employment Research; [3] Cheng, S., Long, J. S. (2007), Testing for IIA in the Multinomial Logit Model. Sociological Methods \& Research, 35(4), 583-600;

[4] Chlosta, S., Patzelt, H., Klein, S. B., Dormann, C. (2012), Parental Role Models and the Decision to Become Self-Employed: The Moderating Effect of Personality. Small Business Economics, 38(1), 121-138;

[5] Corak, M., Piraino, P. (2011), The Intergenerational Transmission of Employers. Journal of Labor Economics, 29(1), 37-68.;

[6] Dunn, T., Holtz-Eakin, D. (2000), Financial Capital, Human Capital and the Transition to Self-employment: Evidence from Intergenerational Links. Journal of Labor Economics, 18(2), 282-305;

[7] Frank, S. A., Lewis, G. B. (2004), Government Employees: Working Hard or Hardly Working? ; American Review of Public Administration,34(1), 36-51;

[8] Fukuyama, F. (1996), Trust: The Social Virtues and the Creation of Prosperity; New York: Free Press/Penguin Books;

[9] Gevrek, D., Gevrek, E. Z. (2010), Nepotism, Incentives and the Academic Success of College Students .Labour Economics, 17(3), 581-591;

[10] Hausman, J., McFadden, D. (1984), Specification Tests for the Multinomial Logit Model . Econometrica, 52(5), 1219-1240;

[11] Houston, D. J., Cartwright, K. E. (2017), Spirituality and Public Service. Public Administration Review; January/February, 88-102;

[12] Judge, T., Martocchio, J., Thoresen, C. (1997), Five-Factor Model of Personality and Employee Absence; Journal of Applied Psychology, 82(5), 745-755;

[13] Lee, S. M., Lee, B. (2015), Entrepreneur Characteristics and the Success of Venture Exit: An Analysis of Single-Founder Start-ups in the U.S. International Entrepreneurship and Management Journal, 11(4), 891-905;

[14] Long, S. J., Freese, J. (2006), Regression Models for Categorical Dependent Variables Using Stata, $2^{\text {nd }}$ Edition. College Station. TX: Stata Press; [15] Lupea, A., Ion, M., Matei, M. Ichim, S. (2016), Barometrul Antreprenoriatului Românesc. Sinteza rezultatelor editiei 2016. Raiffeisen Bank and Ernst\&Young. Available at http://cursdeguvernare.ro/wp- 
Determinants of Entrepreneurial Proclivity of Students in Economics and Business Administration

content/uploads/2016/

05/EY_Barometrul-antreprenoriatului-romanesc_2016_sinteza.pdf;

[16] Matei, M., Strat, A. V., Anagnoste, S., Davidescu, A. A., Zăinescu, A. S., Rădulescu, A. (2014), Barometrul educatiei si culturii antreprenoriale în rândul tinerilor, România 2014. Ernst\&Young, Available at

http://files.finantare.ro/2014/studiu-EY-barometrul-educatiei-si-culturiiantreprenoriale.pdf;

[17] Neville, F., Orser, B., Riding, A., Jung, O. (2014), Do Young Firms Owned by Recent Immigrants Outperform Other Young Firms? . Journal of Business Venturing, 29(1), 55-71;

[18] Njoku, E. C., Ebeh, R. E., Mbaeri, S. O. (2017), Personality Traits as Predictors of Organizational Commitment among Public and Private Sector Employees in Enugu, Nigeria . British Journal of Psychology Research, 5(2), 9-23;

[19] North, D., Thomas, R. P. (1973), The Rise of the Western World: A New Economic History. Cambridge: Cambridge University Press;

[20] Palagashvili, L. (2015), Entrepreneurship, Institutions, and Economic Prosperity, in: Boudreaux, D.J. (ed.), "What America's Decline in Economic Freedom Means for Entrepreneurship and Prosperity", Canada: Fraser Institute, 3-35;

[21] Peterson, L. E. (2010), MLOGITROC: Stata Module to Calculate Multiclass ROC Curves and AUC from MNL Regression. Available at https://EconPapers.repec.org/RePEc:boc:bocode:s457181;

[22] Rauch, A., Frese, M. (2007), Let's Put the Person Back into

Entrepreneurship Research: A Meta-analysis on the Relationship between Business Owners' Personality and Business Creation and Success . European Journal of Work and Organizational Psychology, 16(4), 353-385;

[23] Rothmann, S., Coetzer, E. (2003), The Big Five Personality Dimensions and Job Performance. SA Journal of Industrial Psychology, 29(1), 68-74;

[24] Small, K., Hsiao, C. (1985), Multinomial Logit Specification Tests. International Economic Review, 26(3), 619-627;

[25] Train, K. (2003), Discrete Choice Methods with Simulation. New York: Cambridge University Press.

DOI: 10.24818/18423264/52.3.18.07 